\title{
A thermodynamically consistent constitutive model for diffusion-assisted plasticity in Ni-based superalloys
}

\author{
D. Barba ${ }^{a}$,E. Alaborta ${ }^{\mathrm{a}}$, D. Garcia-Gonzalez ${ }^{\mathrm{a}}$, J.J. Moverare ${ }^{\mathrm{c}}$, R.C. Reed ${ }^{\mathrm{a}, \mathrm{b}}$, \\ A. Jérusalem ${ }^{\mathrm{a}, *}$ \\ ${ }^{a}$ Department of Engineering Science, University of Oxford, Parks Road, Oxford, OX1 3PJ, \\ United Kingdom \\ ${ }^{b}$ Department of Material Science, University of Oxford, Parks Road, Oxford, OX1 3PH, \\ United Kingdom \\ ${ }^{c}$ Division of Engineering Materials, Department of Management and Engineering, \\ Linköping University, SE-58183 Linköping, Sweden
}

\begin{abstract}
An elasto-viscoplastic thermodynamically consistent constitutive model for diffusion-assisted phase transformations is presented here. The model accounts for the different deformation mechanisms, their time dependence, the crystal rotations produced by microtwin propagation and the chemistry-plasticity coupling occurring at high temperature. It is applied to the study of the chemically assisted microtwinning observed in Ni-based superalloys in the temperature range of $600-800^{\circ} \mathrm{C}$. The model parameters are calibrated against multi-directional mechanical data from tensile creep tests of single crystal superalloy MD2. The constitutive model is implemented into a crystal plasticity finite element code to study the activation of the different deformation mechanisms within single crystal and polycrystalline aggregates. A relation between the rotations of the crystal and the creep life of the different crystal orientations is established. The results reveal the critical role of the strong anisotropy of microtwin formation on the asymmetric behavior of the alloy and its relevant role on the mechanical performance.
\end{abstract}

Keywords: creep, crystal plasticity, Ni-based superalloys, phase-transformation

\footnotetext{
${ }^{*}$ Corresponding author

Email address: antoine.jerusalem@eng.ox.ac.uk (A. Jérusalem)
}

Preprint submitted to International Journal of Plasticity

December 14, 2017 
$\Omega_{0}, \bar{\Omega}, \overline{\bar{\Omega}}, \Omega \quad$ initial, first intermediate, second intermediate relaxed and deformed configurations

$\nabla_{\mathbf{X}}$
$\nabla_{\mathbf{x}}$
$e_{0}, e$
$\eta_{0}, \eta$
$q$
$\psi_{0}, \psi$
$\psi_{0}^{\mathrm{pa}}$
$\psi_{0}^{\mathrm{tr}}$

$\psi_{0: \text { chem }}$

$\psi_{0: \text { mech }}$

$\mathbf{I}$

$J$

$J^{\operatorname{tr}}$

$\mathbf{F}$

$\mathbf{F}^{\mathrm{e}}$

$\mathbf{F}^{\mathrm{p}: \mathrm{s}}$

$\mathbf{F}^{\mathrm{p}: \operatorname{tr}}$

V

1

$1^{\mathrm{e}}$

$\mathbf{L}_{0}^{\mathrm{p}: \mathrm{s}}, \overline{\overline{\mathbf{L}}}^{\mathrm{p}: \mathrm{s}}$

$\mathbf{L}_{0}^{\mathrm{p}: \operatorname{tr}}, \overline{\mathbf{L}}^{\mathrm{p}: \operatorname{tr}}$

$\mathbf{d}^{\mathrm{e}}$

$\overline{\overline{\mathbf{D}}}^{\mathrm{p}: \mathrm{s}}$

$\overline{\mathbf{D}}^{\mathrm{p}: \operatorname{tr}}$

$\overline{\overline{\mathbf{W}}}^{\mathrm{p}: \mathrm{s}}$

$\overline{\mathbf{W}}^{\mathrm{p}: \operatorname{tr}}$

$\boldsymbol{\sigma}$

$\sigma_{\mathrm{pa}}$

$\boldsymbol{\sigma}_{\mathrm{tr}}$

$\mathbf{S}_{0}, \overline{\mathbf{S}}, \overline{\overline{\mathbf{S}}}$

$\overline{\overline{\mathbf{M}}}$

$T$

$p$

ume in $\Omega_{0}$ and $\Omega$

specific entropy per unit volume in $\Omega_{0}$ and $\Omega$

heat flux per unit volume in $\Omega$

Helmholtz free energy per unit volume in $\Omega_{0}$ and $\Omega$

parent-phase Helmholtz free energy per unit volume in $\Omega_{0}$

transformed-phase Helmholtz free energy per unit volume in $\Omega_{0}$

chemical term of the specific

Helmholtz free energy per unit volume in $\Omega_{0}$

mechanical term of the specific

Helmholtz free energy per unit volume in $\Omega_{0}$

identity matrix

determinant of the deformation gradient

determinant of the transformation deformation gradient

deformation gradient tensor

elastic deformation gradient tensor

plastic slip deformation gradient

tensor

plastic transformation deformation gradient tensor

velocity tensor

velocity gradient tensor

elastic velocity gradient tensor

plastic slip velocity gradient tensor in $\Omega_{0}$ and $\overline{\bar{\Omega}}$

plastic transformation velocity gradient tensor in $\Omega_{0}$ and $\bar{\Omega}$

symmetric part of the elastic velocity gradient tensor in $\Omega$

symmetric part of the plastic slip velocity gradient tensor in $\overline{\bar{\Omega}}$

symmetric part of the plastic transformation velocity gradient tensor in $\bar{\Omega}$

skew part of the plastic slip velocity gradient tensor in $\overline{\bar{\Omega}}$

skew part of the plastic transformation velocity gradient tensor in $\bar{\Omega}$

Cauchy stress tensor

parent phase Cauchy stress tensor

transformed phase Cauchy stress tensor

second Piola-Kirchhoff stress tensor in $\Omega_{0}, \bar{\Omega}$ and $\overline{\bar{\Omega}}$

Mandel stress tensor in $\overline{\bar{\Omega}}$

absolute temperature

pressure
C
$\mathbb{C}$

$\mathbb{C}_{\text {crys }}$

$\mathbf{R}_{\text {crys } \rightarrow \Omega_{0}}$

$\mathbf{R}_{\text {twin }}$

elastic right Cauchy-Green tensor

elastic stiffness matrix in $\Omega_{0}$

elastic stiffness matrix in the crystal reference frame

rotation matrix between crystal reference frame and $\Omega_{0}$

rotation matrix between the parent crystal reference frame and the twin crystal reference frame

$\overline{\overline{\mathbf{m}}}$

$\overline{\overline{\mathbf{n}}}$

slip direction

slip plane normal

condensed deformation vector associated with the phase transformation

$\gamma_{\alpha}$

$f_{0}$

$f$

$f_{0: \beta}$

$\delta_{\mathrm{tr}: \beta}$

$\gamma_{\operatorname{tr}}$

$\mathbf{F}_{\Delta V}$

$m_{\alpha / \beta}$

$\tau$

$s_{\alpha / \beta}$

$\dot{\gamma}_{0}$

$c_{\mathrm{tr}}$

$c_{\text {pa }}$

$\mu_{\mathrm{tr}}$

$\mu_{\mathrm{pa}}$

G

$p$

$\mathcal{J}$

$T$

$\dot{D}$

$\dot{D}_{\text {slip }}$

$\dot{D}_{\text {transformation }}$

$\dot{D}_{\text {diffusion }}$

$\Gamma_{S F}$

$a$

A

$\phi$

$h$

$q$ shear strain of slip system $\alpha$ volumetric transformed phase fraction in $\Omega_{0}$

volumetric transformed phase fraction in $\Omega$

transformed fraction of system $\beta$ in $\Omega_{0}$

transformation dilatational strain per unit volume of system $\beta$

transformation shear strain per unit volume

volumetric deformation gradient associated with the phase transformation produced per unit volume of slip system $\alpha$

rate sensitivity parameter of system $\alpha / \beta$

resolved shear stress

resistance for slip/phase transformation of system $\alpha / \beta$

reference strain rate

chemical concentration in the transformed phase in $\Omega_{0}$

chemical concentration in the parent phase in $\Omega_{0}$

specific chemical potential of the transformed phase in $\Omega_{0}$

specific chemical potential of the parent phase in $\Omega_{0}$

specific free Gibbs energy

pressure

chemical flux

temperature

global dissipation rate

dissipation rate due to slip deformation

dissipation rate due to phase transformation

dissipation rate due to diffusion between phases

stacking fault energy per unit area of fault

lattice parameter

area of stacking fault

plastic function

hardening moduli parameter

interaction hardening parameter 
dissipation rate due to slip deformation in $\bar{\Omega}$

$\dot{\overline{\bar{D}}}_{\text {transformation }}$

dissipation rate due to phase transformation in $\overline{\bar{\Omega}}$ 


\section{Introduction}

Ni-based superalloys are a class of materials widely used in the aerospace industry due to their outstanding properties at high temperatures. Their creep deformation behavior controls the service life of most turbine components in modern aeroengines [1]. The in-service micromechanics of these materials consists of precipitation hardening of the $\gamma^{\prime}$ phase $\left(L 1_{2}\right.$ crystal structure) embedded coherently in the $\gamma$ face-centred cubic (FCC) matrix [2]. Although their strong dependence upon temperature, orientation and the magnitude of the applied stress is well known [1, their micromechanics is not entirely understood. Driven by efficiency improvements, the temperature and stress conditions in service are now leading the alloy to perform in the range of $700-850^{\circ} \mathrm{C}$. These new loading scenarios results in new deformation mechanisms that affect the mechanical performance of the material [3, 4].

The plastic deformation of superalloys at low temperatures $\left(T<700^{\circ} \mathrm{C}\right)$ is governed by the shearing of the $\gamma^{\prime}$ precipitates mediated by high-energy faults which considerably strengthen the alloy $[2,5]$. In this temperature regime, the diffusion processes are still limited and the dislocations are solely assisted by the driving force provided by the external stress. Above this temperature regime $\left(T>700^{\circ} \mathrm{C}\right)$, the governing deformation mechanisms are characterized by a complex coupling between diffusion and plastic deformation [6, 7. During these processes, the dislocation shearing within the $\gamma^{\prime}$ precipitates is supported by the segregation of $\mathrm{Co}$ and $\mathrm{Cr}$ elements at the faults created [3, 8]. This considerably reduces the stress required to deform the precipitates and causes a drop in the performance of the material [3, 9, 8, 2]. These forms of plasticity include microtwinning shearing [4, 10, extrinsic stacking faults [11, 3] and, lately reported, the antiphase boundary slip [8]. These diffusion processes are at the source of relevant plastic events: anomalous yielding, a substantial strain 
hardening effect, an extensive anisotropy and a tensile/compressive asymmetric behavior that does not follow the Schmid theory [12, 13, 14].

The complex behavior of superalloys in the studied range of temperatures $\left(700-850^{\circ} \mathrm{C}\right)$ makes it challenging to predict their mechanical response and performance, as required for optimizing the efficiency of components design. Therefore, reliable models able to describe the different aspects of high temperature deformation, including the effect of the chemical diffusion processes, are of both theoretical and practical interests.

Crystal plasticity (CP) has demonstrated its ability to faithfully model the mechanical crystalline response of superalloys at high temperatures [15, 16, 17. 18. Ghosh et al. [15] proposed a phenomenological CP model for uniaxial creep of single crystal superalloys including the effect of crystallography and tertiary creep. In this approach, slips over octahedral $\{111\}$ and cubic $\{001\}$ planes are considered. Brehm et al. 19] extended this study by incorporating a precise description of the dislocation densities for the different slip systems and their interactions. MacLachan et al. [16] proposed a simplified power-law model with a reduced number of parameters that incorporates the effect of partial $\langle 112\rangle$ shear on the creep rates, which was assumed to be symmetric. Their predictive results are in good agreement with the creep response in tensile $\langle 001\rangle$ and $\langle 111\rangle$ orientations. The work of Busso et al. 20] accounts for the additional hardening effects associated with dislocation density gradients, which have been proved crucial to cause the strengthening in superalloys. Part of this work is further extended in Zhao et al. [21] in a diffusion-mechanical coupled framework to study the influence of oxidation on the crack initiation in Ni-based superalloys CMSX-4. The work of Kashiro et al. 22, provides a detailed insight into the nucleation mechanisms of $\langle 110\rangle$ superpartials using a coupled finite element model (FEM)-dislocation dynamics model. Shenoy et al. [23. developed a rate 
dependent, microstructure-sensitive $\mathrm{CP}$ model of the mechanical behavior of polycrystalline IN100 at $650^{\circ} \mathrm{C}$. The model is based on the generation of representative volume elements (RVEs) of the actual microstructure and a physically based CP constitutive framework. Ma et al. 18 incorporated the climb mechanism over $\gamma^{\prime}$ precipitates in a dislocation-based CP model and obtained a good prediction of all stages of creep for $\langle 001\rangle$ orientation. They further developed the model by including the chemical dependency of the diffusivity on the climbing rates 24. The work of Kim et al. 25] extended this approach for a wider range of alloys by accounting for the alloy composition in the calculation of the elastic properties. Vorster et al. 26] developed a RVE method for predicting the creep strength of a wide range of single crystal superalloys. In this model, the plastic deformation (in the form of a stochastic viscous law) is constrained to the $\gamma$ channels, while the $\gamma^{\prime}$ precipitates are assumed to deform elastically. This method has been extended by Keshavarz et al. in several studies to a three-scale framework [27, 28, 29, 17]: (i) precipitate level, (ii) single crystal level, (iii) polycrystalline level. The bridge among scales is performed by the identification of the material parameters from the lower scale in a bottom-up approach. In this model they have included the plastic deformation of the $\gamma^{\prime}$ phase and the effect of phenomena not following Schmid theory. The major limitation of the models developed until now is that their effectiveness is usually proven for a limited set of crystal orientations or loading conditions without accounting for the chemical changes during plastic deformation reported in the literature [4, 3, 10].

Despite the abundant amount of research on the mechanical behavior of superalloys at high temperature under creep, only a few studies focus on chemical changes during plastic deformation. These studies are usually limited to experimental reports of their existence [10, 30, 31]. Paradoxically, these changes are 
not compatible with the classical strengthening mechanism of high-energy fault shearing of $\gamma^{\prime}$ precipitates [3, 8]; thus they are crucial for the creep strength of the alloy. This consideration is especially important above $700^{\circ} \mathrm{C}$ where the diffusion processes during deformation become dominant. A chemical-mechanical bridge between physical modeling at the mechanistic lower scale and the continuum modeling at the higher scale is thus needed.

The work presented here is structured as follows: in Section 2 , post-mortem experimental observations of a prototype single crystal superalloy are first presented to define the phase transformation problem. The general continuum modeling framework for phase transformations is then presented in Section 3 . This framework is developed for finite deformation within a thermodynamically consistent framework. The model couples the mechanics of the phase transformation with changes of the chemical composition and, therefore, diffusion between phases. The diffusion problem is then particularized for creep microtwinning plasticity in Ni-based superalloys. The diffusion phase transformation theory is derived from the previous work of Barba et al. 4]. The model is then used to describe the orientation dependent behavior of the prototype single crystal superalloy MD2 in Section 4. Finally, the model is extended in Section 5 to study the simultaneous activities of different plastic mechanisms in a polycrystalline aggregates and their influence on the mechanical behavior at high temperatures. Both single crystal and polycrystal applications demonstrate the critical role of the diffusion-assisted microtwinning mechanism in providing the high antisymmetric behavior observed in superalloys.

\section{Experimental creep campaign}

The prototype single crystal Ni-based superalloy MD2 was used in this study. Its chemical composition is presented in Table 1. The material was solution 
treated at $1275^{\circ} \mathrm{C}$ for 8 hours, followed by 6 hours of ageing at $1080^{\circ} \mathrm{C}$ and finally at $870^{\circ} \mathrm{C}$ for 16 hours.

\subsection{Mechanical behavior}

A series of tension and compression uniaxial creep tests at $800^{\circ} \mathrm{C}$ were performed. This temperature was selected for its previously reported prevalence of microtwinning 4]. Three different high-symmetry orientations were tested: $\langle 001\rangle,\langle 110\rangle$ and $\langle 111\rangle$. Electron-backscattered diffraction (EBSD) was used to measure the deviation from the ideal nominal orientation leading to the crystal orientations given in Table 2, The testing conditions for all tests are presented in Table 3 .

An overview of the tested specimens geometries is presented in Fig. 1. Tensile tests were conducted on dog bone specimens of $1.6 \times 1 \mathrm{~mm}^{2}$ cross-sectional area using an Instron electrothermal-mechanical testing machine. Cylinders of $5 \mathrm{~mm}$ diameter and $5 \mathrm{~mm}$ height were employed for the compression tests in an Instron servo-electric machine. Digital image correlation was used to track the material deformation for all tests. The measured tensile and compression behaviors are shown in Fig. 2. A strong anisotropy and a tension-compression asymmetric behavior is observed, especially for the crystals oriented along $\langle 011\rangle$ and $\langle 001\rangle$ directions. This behavior is in agreement with previous work in the literature for creep in Ni-based superalloys [16, 9, 32. Further examination of the active deformation mechanisms is presented next.

\subsection{Microtwinning shearing in Ni-based superalloys}

A post-mortem examination of the samples was carried out to identify the orientations and conditions for which microtwinning occurred. Scanning electron microscopy (SEM) and EBSD techniques were used to identify the deformation features and confirm their twin character. Some examples of these 
Table 1: Chemical composition (wt.\% and at.\%) of Ni-based superalloy MD2.

\begin{tabular}{lcccccccccc}
\hline & $\mathrm{Ni}$ & $\mathrm{Al}$ & $\mathrm{Co}$ & $\mathrm{Cr}$ & $\mathrm{Hf}$ & $\mathrm{Mo}$ & $\mathrm{Ta}$ & $\mathrm{Si}$ & $\mathrm{Ti}$ & $\mathrm{W}$ \\
\hline wt. \% & Bal. & 5.0 & 5.1 & 8.0 & 0.1 & 2.1 & 6.0 & 0.1 & 1.3 & 8.1 \\
at. \% & Bal. & 11.24 & 5.25 & 9.33 & 0.03 & 1.33 & 2.01 & 0.22 & 1.65 & 2.63 \\
\hline
\end{tabular}


Table 2: Nominal orientations and measured orientations for the single crystal samples used in this study.

\begin{tabular}{|c|c|c|c|}
\hline tion & $\left\langle\begin{array}{lll} & 0 & 1\end{array}\right\rangle$ & $\left\langle\begin{array}{llll}1 & 1 & 0\end{array}\right.$ & $\left.\begin{array}{llll}1 & 1 & 1\end{array}\right\rangle$ \\
\hline Orienta & $\langle 0.02-0.07 \quad 0.99\rangle$ & $\langle 0.730 .67-0.08\rangle$ & $\begin{array}{llll}0.66 & 0.53 & 0.54\rangle\end{array}$ \\
\hline
\end{tabular}


Table 3: Creep tested conditions for single crystal MD2.

\begin{tabular}{|c|c|c|c|c|c|c|c|c|}
\hline Direction & \multicolumn{4}{|c|}{ Tension (MPa) } & \multicolumn{4}{c|}{ Compression (MPa) } \\
\hline$\langle 001\rangle$ & - & 650 & 675 & 700 & 625 & 650 & 675 & 700 \\
\hline$\langle 011\rangle$ & 625 & 650 & 675 & 700 & - & 650 & - & 700 \\
\hline$\langle 111\rangle$ & - & 650 & - & 700 & - & 650 & - & 700 \\
\hline
\end{tabular}




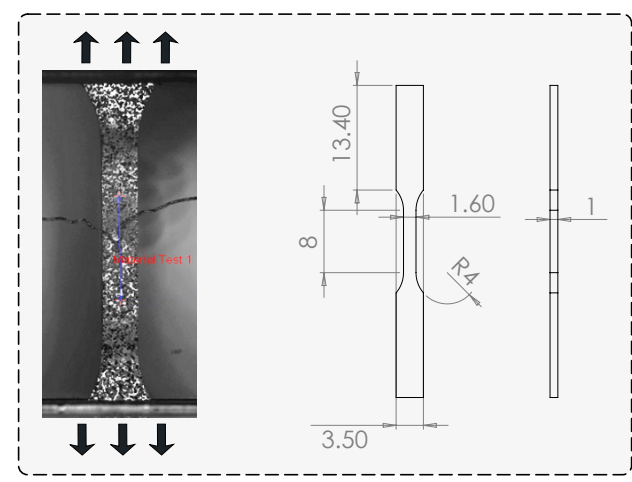

Tension

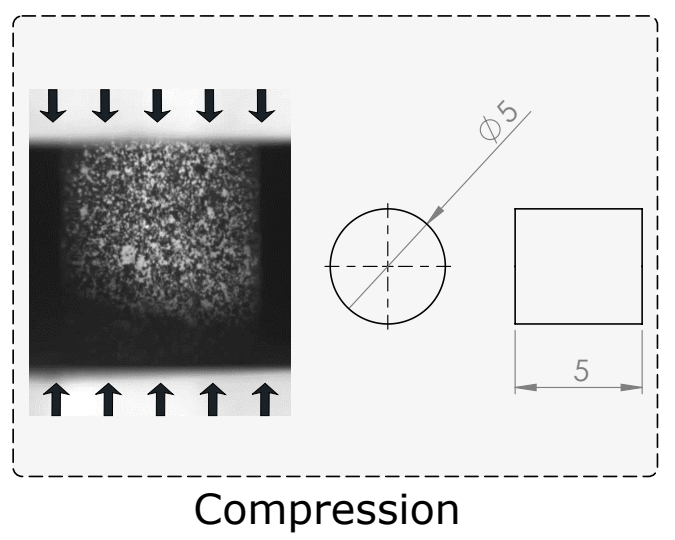

Figure 1: Experimental setup and samples geometry used for tensile and compressive creep tests (measurements are in $\mathrm{mm}$ ). Patterns for DIC measurements are shown in both cases. 

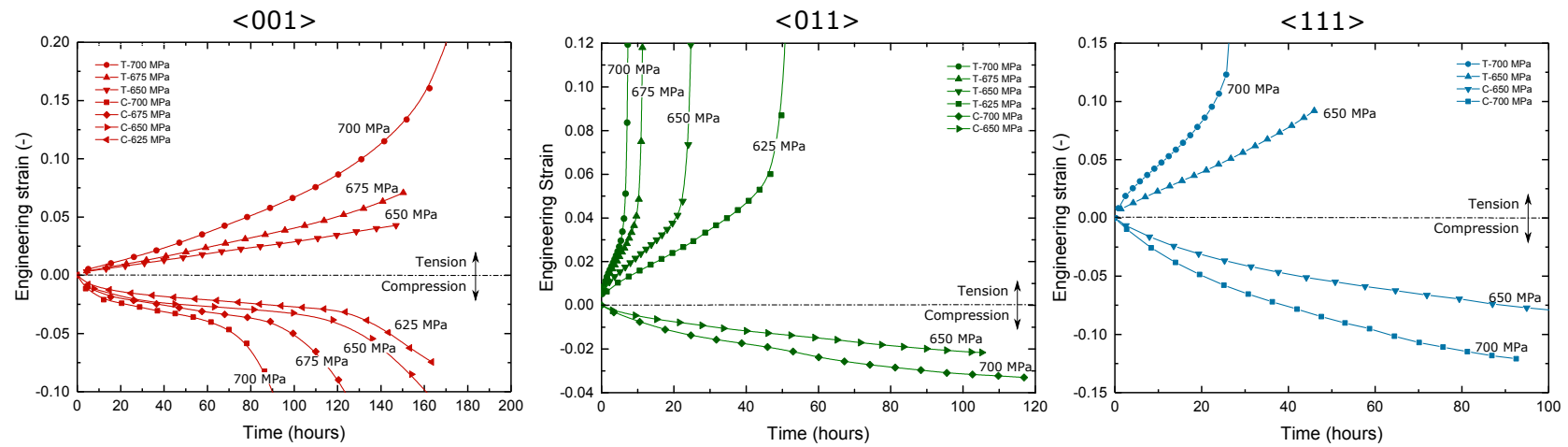

Figure 2: Experimental creep curves for MD2 single crystal superalloy at $800^{\circ} \mathrm{C}$ for the loading directions $\langle 001\rangle,\langle 011\rangle$ and $\langle 111\rangle$ at stresses between $625 \mathrm{MPa}$ and $700 \mathrm{MPa}$. T stands for tension and $\mathrm{C}$ stands for compression. 
deformed structures are presented in Fig. 3a. The activation of microtwins was observed along the $\langle 011\rangle$-direction during tensile deformation and along the $\langle 001\rangle$-direction in compression. The number of active twin systems was found to vary from one direction to another. A single twin system was identified for tensile $\langle 011\rangle$ microtwins while for $\langle 001\rangle$ compression microtwins, two conjugate families of twin systems were observed. This is in agreement with the number of high stress twin systems for the measured orientations. The fact that these twin bands formation was not observed in the reversed load tests ( $\langle 001\rangle$-compression and $\langle 011\rangle$-tension) implies that the microtwinning formation mechanism is strongly antisymmetric.

The different deformation mechanisms responsible for this behavior are presented in the atomic model of Fig. 3 3 b. In this figure, three adjacent FCC closed packed 111 atomic planes are presented. By sliding the top atomic layer in $3 \mathrm{~b}$ over the bottom and middle layers along the closed packed FCC slip directions $\langle 011\rangle$ and $\langle 112\rangle$, the following different deformation mechanisms take place:

- Antiphase boundaries (octahedral slip): Slip along $\langle 011\rangle$ directions by a full dislocation (with a Burgers vector $|\mathbf{b}|=a / \sqrt{2}$, where a is the lattice parameter). This leads to a correct stacking sequence but wrong neighbors structure at the shear plane (the local ordering of $\mathrm{Al}$ and $\mathrm{Ni}$ atoms is corrupted). This structure is called antiphase boundary (APB). This shear direction is symmetric and it cannot explain by itself the mechanical response observed. Hereafter, this mechanism is referred to as octahedral slip.

- Stacking faults and microtwinning: Slip along $\langle 112\rangle$ directions by partial dislocations. In this case, two different situations can happen:

- Microtwinning (or SESFs): The dislocation shear occurs in the directions indicated by red arrows in Fig. $3 \mathrm{p}(|\mathbf{b}|=a \sqrt{6} / 2)$. The 


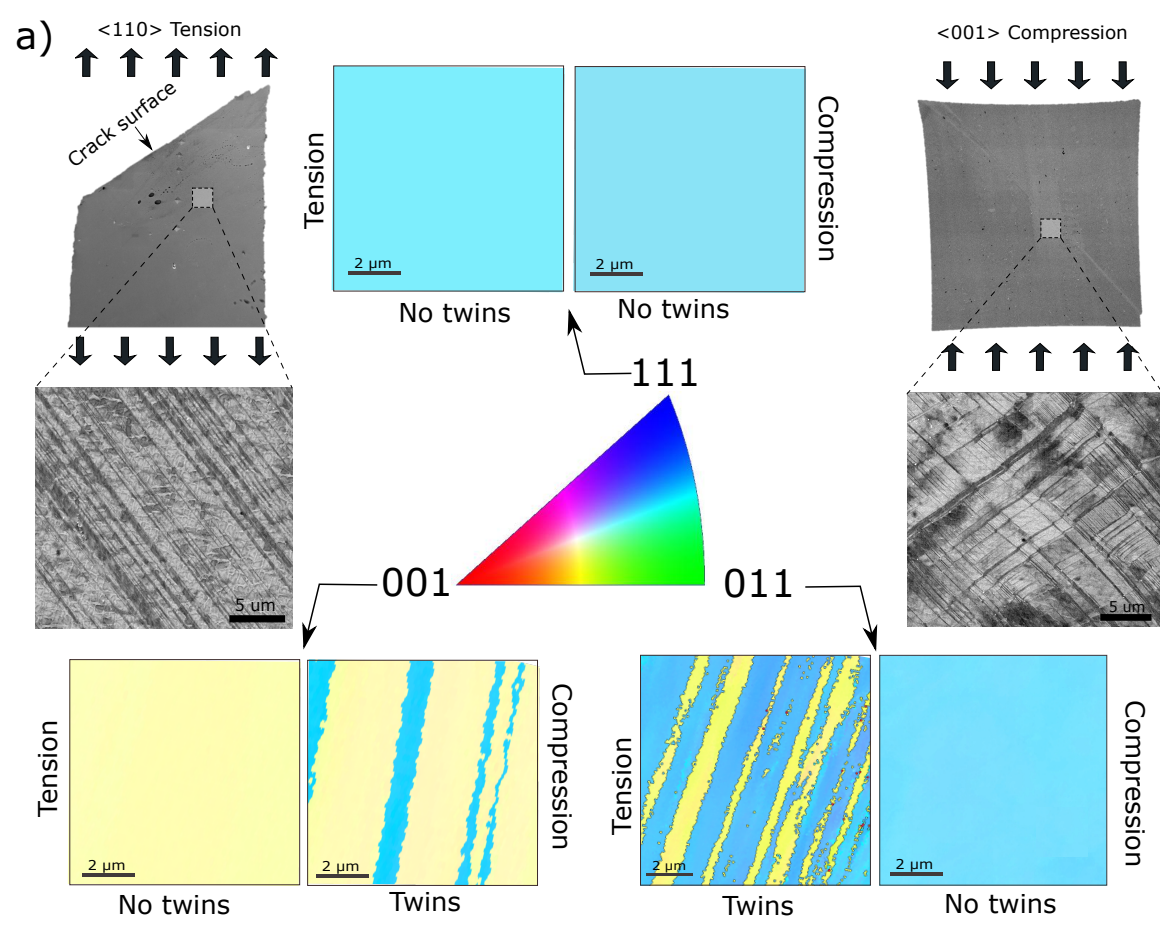

b) Sliding directions and mechanisms in $\gamma_{\text {-phase in Ni-based superalloys }}^{\prime}$
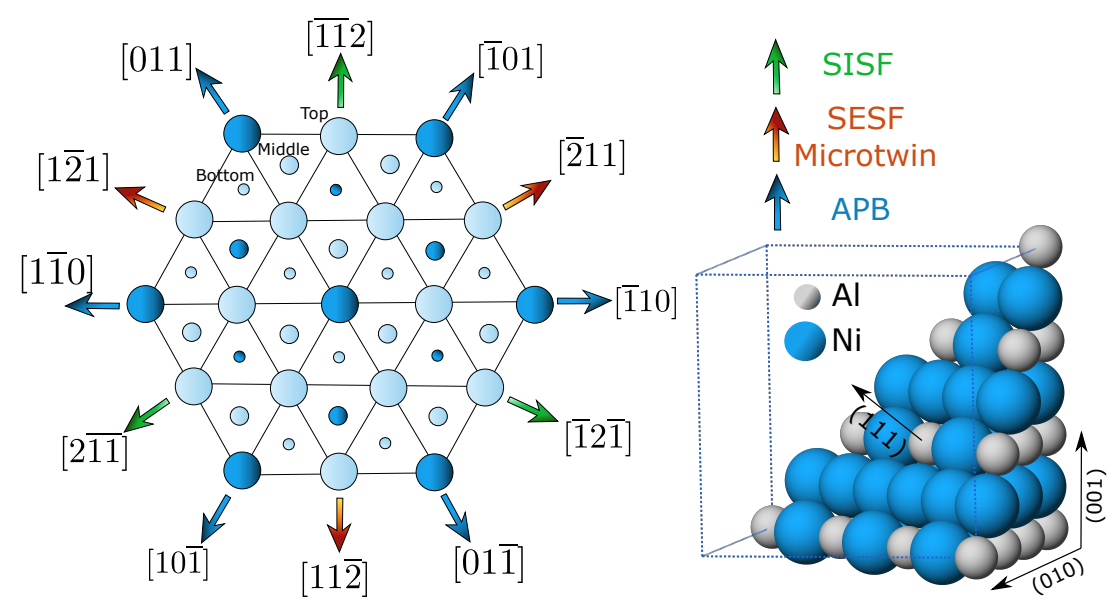

Figure 3: a) EBSD maps of the tested samples at $800^{\circ} \mathrm{C}$ indicating the conditions for which microtwinning is active: $\langle 011\rangle$ in tension and $\langle 001\rangle$ in compression along with experimental micrographies of the $650 \mathrm{MPa}-\langle 011\rangle$-tension and $650 \mathrm{MPa}-\langle 001\rangle$ - compression specimens containing microtwins after testing; b) crystallography of slip deformation along $\{111\}$ planes in Ni-based superalloys. 
a)

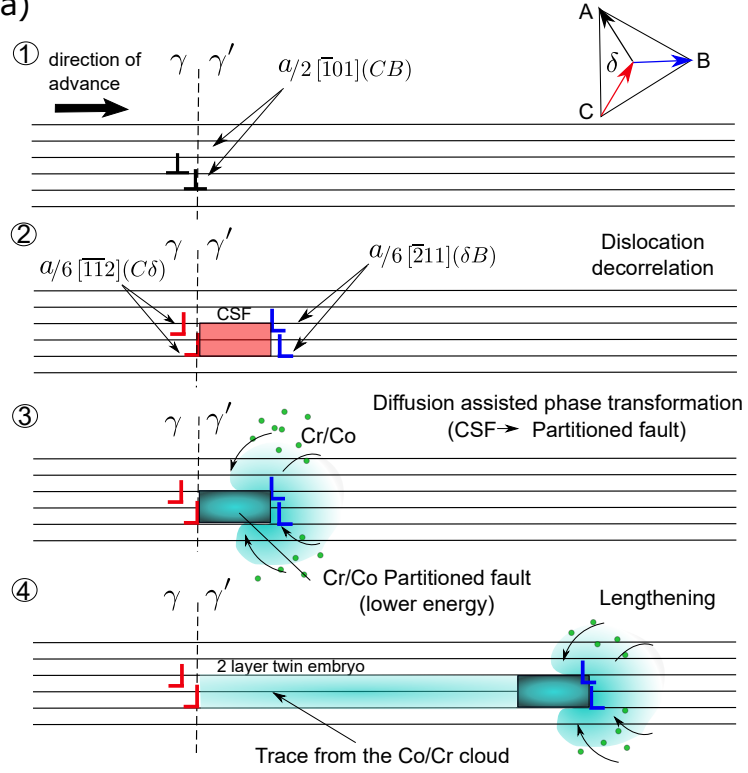

b)

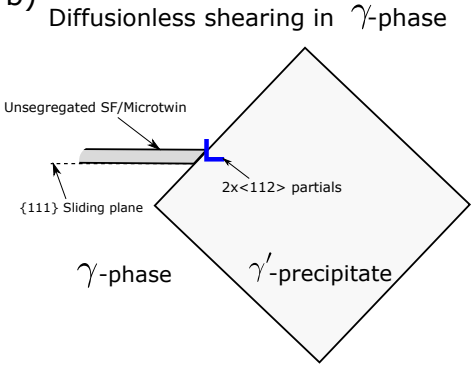

c)

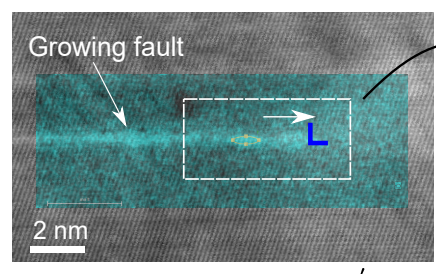

Diffusion assisted shearing in $\gamma^{\prime}$-phase
Diffusion assisted shearing in $\gamma_{\text {-phase }}^{\prime}$
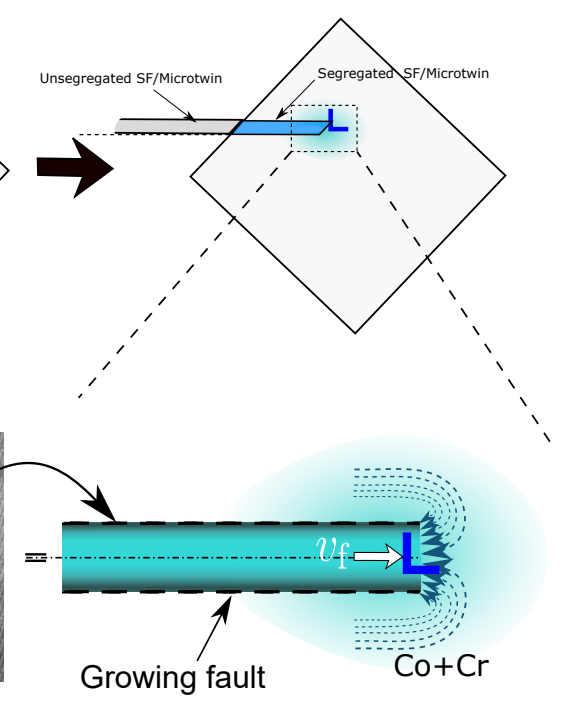

Figure 4: a) Diffusion-assisted plasticity: microtwinning forming process in the precipitate: (1) two perfect a/2 [101] dislocations are trapped at the $\gamma / \gamma$ phase boundary; (2) the full dislocations split into four a/6 [211] partials, the two partials with higher Schmid factor (blue) enter the precipitate creating a high energy CESF thus preventing their propagation; (3) Co and $\mathrm{Cr}$ enrichment at the fault transforms the CESF to a lower energy microtwin embryo; (4) the leading partials continue the shearing of the precipitate and leave traces of Co and $\mathrm{Cr}$ behind them; b) differences between microtwinning formation in (diffusionless shearing) and $\gamma$ (diffusion-assisted plasticity) phases; c) experimental observation of the segregation of $\mathrm{Cr}$ during the microtwin formation. Adapted from Barba et al. 8 . 
shear creates a high energy atomic structure called complex intrinsic stacking fault (CISF) with a wrong atomic order and stacking sequence $\mathrm{ABCACABC}$. The consecutive shear of two dislocations of this type over adjacent atomic layers produces a high energy pseudo microtwin called complex extrinsic stacking fault (CESF) (Fig. 4a2). Dislocations cannot continue shearing because of the high energy fault created. The diffusion of $\mathrm{Co}$ and $\mathrm{Cr}$ to this pseudo microtwin relaxes the wrong atomic structure and transforms this high energy fault to a low energy true microtwin embryo or superlattice extrinsic stacking fault (SESF) [Smith et al. 2016, Freund et al. 2017, Barba et al. 2017] (Fig. 4 -3). The advance of the twin partials is now limited by the segregation of Co and Cr to the growing fault (Fig. 4 4 -4). This implies long range diffusion and, therefore, a time-dependent shearing of the precipitates. In the present work, this is named diffusion-assisted plasticity and, constitutively, is treated as a phase transformation. The stress applied on the dislocation needs to be in equilibrium with the back-stress required to create the new fault or phase. This back-stress is proportional to the energy of the new fault and, therefore, dependent on the degree of phase transformation arising from the segregation of Co and Cr. Additionally, some differences have been reported in the mechanism of microtwinning between $\gamma$ and $\gamma$ phases [33, 10]. Barba et al. 33] and Rae et al. 10] observed that the segregation processes only occur within the $\gamma$ phase; no segregation was found in the $\gamma$ channels. This scenario is schematized in Fig. 4 p. Moreover, Barba et al. proposed that segregation is due to wrong neighbor structures forming around the disordered phase $\gamma$. Therefore, no diffusion of $\mathrm{Co}$ and $\mathrm{Cr}$ is needed in $\gamma$ to lower the energy of 
the faults. This is included in the diffusion model by homogenizing the calculated velocities for diffusion-assisted shearing over the $\gamma$ and $\gamma$ phases.

\section{- Detwinning and superlattice intrinsic stacking fault (SISF):}

The dislocation slip occurs along the $\langle 112\rangle$ directions, represented by green arrows in Fig. 3b $(|\mathbf{b}|=a / \sqrt{2}$ for detwinning and $|\mathbf{b}|=3 a / \sqrt{2}$ for SISF). If a twin is already present in the dislocation plane, two dislocations of $|\mathbf{b}|=a \sqrt{6} / 2$ on adjacent planes would produce a detwinning process which recovers the configuration of the parent crystal. However, detwinning is unlikely to happen under uniaxial loading conditions, and thus is not considered in this work. Moreover, dislocation shear in this direction on non-twinned material is only possible with a Burgers vector size of three times the twinning one $(|\mathbf{b}|=3 a / \sqrt{2})$ this is due to the atomic positions of the lower layer (see Fig. 3b). This kind of shear would produce a SISF structure. However, such a large Burgers vector would need a high elastic energy to form the partial dislocation [34, thus the mechanisms is excluded from the present model. In addition, the atomic path for the sliding event requires to overpass the atoms on the subsequent layer, which involves an extra high-energy barrier, and makes the mechanism even more unlikely.

In the following section, these important aspects of the high temperature deformation of Ni-based superalloys (deformation asymmetry and diffusion assisted shearing) are introduced in a continuum theory. This leads to a phase transformation framework applied later for the study of segregation-assisted plasticity in superalloys. 


\section{Continuum mechanics framework}

As presented in the previous section, the plastic deformation of superalloys at high temperatures (mainly due to stacking faults and microtwinning) is intimately linked with chemical fluctuations during service. These chemical fluctuations are observed within the plastic regions, evolving through a transformation process bearing resemblance to a diffusional phase transition. These findings differ from the old fashioned static view of the plastic flow, in which the general crystal structure (i.e., crystal rotations from twinning is still considered though) and the composition remain unchanged during deformation.

In this work, the irreversible part of the total deformation is assumed to be the combination of:

- Standard plastic slip: the flow rule is decomposed into elemental shear slips over the different slip systems of the crystal. The atomic structure of the material remains unaltered after deformation, and so does its elastic behavior. Two different slip systems are considered herein according to previous works in the literature [15, 17]: $\{111\}$ octahedral and $\{001\}$ cubic slips. Cubic slip is used in crystal plasticity models to capture the effect of octahedral dislocations cross-slip or collinear annihilation between dislocations with the same Burgers vector gliding in different planes [35, 36, 37.

- Phase transformation induced deformation: in a general case, this term can consist not only of shear deformations but also of dilatational contributions associated with the phase transformation. In terms of the material structure, it can either change its atomic ordering (martensitic transformations) or its orientation (twinning). Generally, these change the elastic resistance of the material. With regards to the specific case treated in this manuscript, segregation assisted microtwinning is considered as a diffu- 
Table 4: Slip and microtwin systems used in the crystal plasticity framework

\begin{tabular}{|c|c|c|}
\hline Octahedral Slip & Cubic Slip & Microtwinning \\
\hline$\pm(111)-[10 \overline{1}]$ & $\pm(001)-[110]$ & $+(111)-[11 \overline{2}]$ \\
\hline$\pm(111)-[1 \overline{1} 0]$ & $\pm(001)-[1 \overline{1} 0]$ & $+(111)-[1 \overline{2} 1]$ \\
\hline$\pm(111)-[0 \overline{1} 1]$ & $\pm(100)-[0 \overline{1} 1]$ & $+(111)-[\overline{2} 11]$ \\
\hline$\pm(\overline{1} 11)-[101]$ & $\pm(100)-[011]$ & $+(\overline{1} 11)-[\overline{1} 1 \overline{2}]$ \\
\hline$\pm(\overline{1} 11)-[110]$ & $\pm(010)-[101]$ & $+(\overline{1} 11)-[\overline{12} 1]$ \\
\hline$\pm(\overline{1} 11)-[0 \overline{1} 1]$ & $\pm(010)-[101]$ & $+(\overline{1} 11)-[211]$ \\
\hline$\pm(1 \overline{1} 1)-[10 \overline{1}]$ & & $+(1 \overline{1} 1)-[1 \overline{12}]$ \\
\hline$\pm(111)-[110]$ & & $+(111)-[121]$ \\
\hline$\pm(1 \overline{1} 1)-[011]$ & & $+(1 \overline{1} 1)-[\overline{21} 1]$ \\
\hline$\pm(11 \overline{1})-[101]$ & & $+(11 \overline{1})-[1 \overline{21}]$ \\
\hline$\pm(111)-[1 \overline{1} 0]$ & & $+(11 \overline{1})-[211]$ \\
\hline$\pm(11 \overline{1})-[011]$ & & $+(11 \overline{1})-[112]$ \\
\hline
\end{tabular}

sional phase transformation that produces a reorientation of the lattice and no dilatation.

The model is represented by the rheological scheme shown in Fig. 5. The different deformation mechanisms are represented by three nonlinear viscous dashpots capturing the rate dependency of each mechanism. In the following, we adopt subscripts and superscripts 'pa' and 'tr' to indicate parent phase and transformed phase of the body, respectively. The different slip and twinning systems are presented in Table 4 .

Furthermore, we assume that the parts of the body undergoing a phase transformation only deform elastically once the transformation occurs. The Cauchy stress $\boldsymbol{\sigma}$ of the homogenized body is the sum of the two phases contributions, i.e., the parent phase resistance $\boldsymbol{\sigma}_{\mathrm{pa}}$ and the transformed phase resistance $\boldsymbol{\sigma}_{\mathrm{tr}}$ :

$$
\boldsymbol{\sigma}=\boldsymbol{\sigma}_{\mathrm{pa}}+\boldsymbol{\sigma}_{\mathrm{tr}}
$$

Both stress tensors are characterized by linear elastic springs in Fig. 5. Their corresponding micromechanical phase activators control the contribution of each phase to the total stiffness as the material undergoes the phase transformation. 


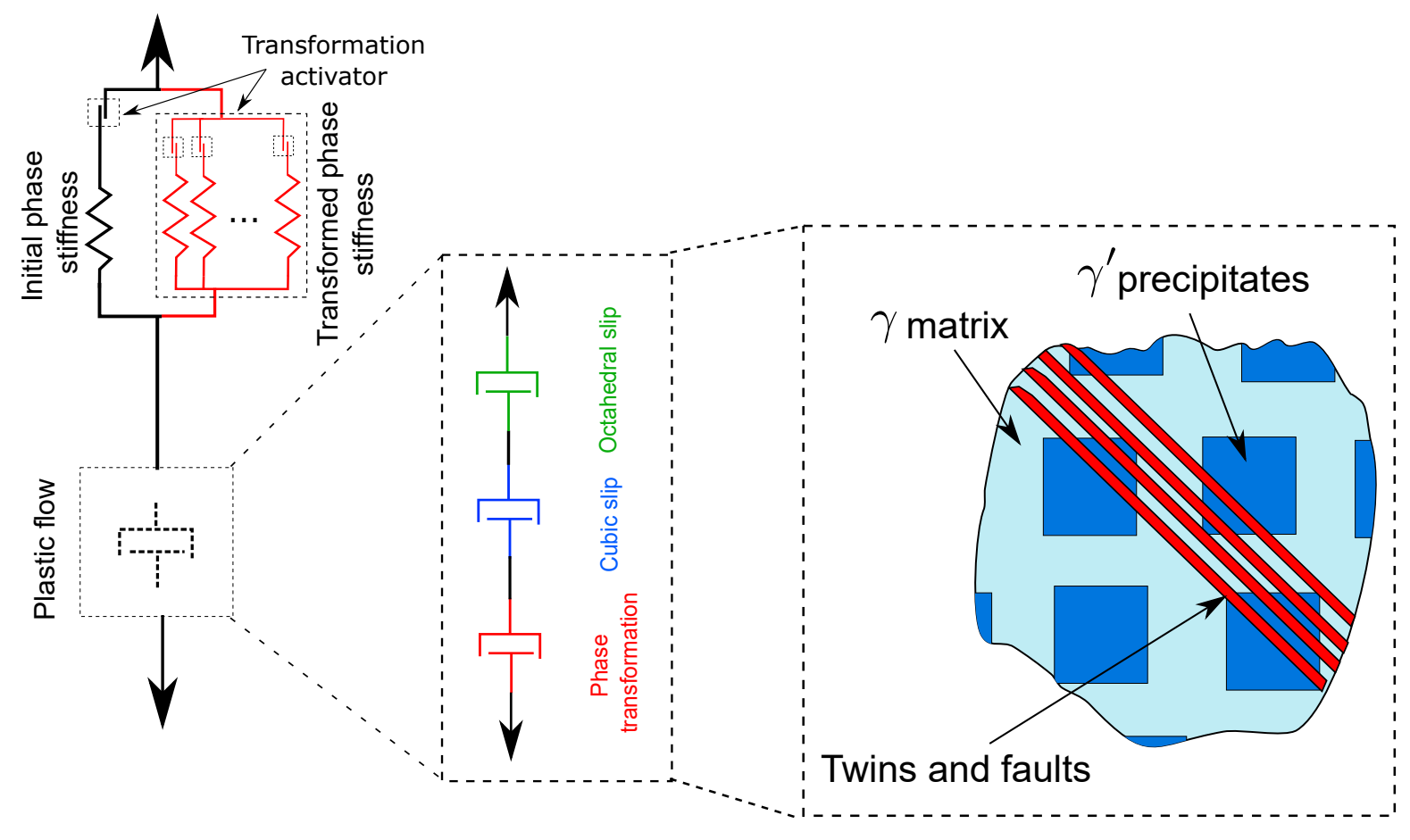

Figure 5: Rheological scheme of the proposed constitutive model and microstructural diagram of a microtwinned structure for Ni-based superalloys. 
The formation of stacking faults and microtwins has been observed to be triggered by local chemical changes 4, 3. More specifically, the enrichment of $\mathrm{Co}$ and $\mathrm{Cr}$ in the new phase lower substantially its formation energy, and thus the required stress for the transformation. The dependence of the phase deformation resistance on $\mathrm{Co}$ and $\mathrm{Cr}$ concentration is included in the model through the definition of the free energy density of the new phase. This concentration is defined in the diffusion-assisted transformation problem. The solution to this diffusional problem between both phases has been already presented in the work of Barba et al. 4 and is used here within a continuum mechanics framework. In the present work, the heat terms are neglected as the body is assumed to deform under isothermal conditions. This assumption is consistent with the small heat produced by plastic deformation with respect to the thermal energy stored in the body at high temperatures. Heat terms can be incorporated trivially if needed.

It is noteworthy that the phase transformation theory described here can be also applied to other physical problems such as martensitic phase transitions in steels [38, 39] or growth problems in biological tissues [40, 41] where the material structure evolves with time and, therefore, the properties also change. In the next section, the kinematics, thermodynamics and constitutive details of the model are presented in detail.

\subsection{Kinematics}

Four configurations are considered to define the kinematics of the model, starting from an initial reference configuration $\Omega_{0}$ to a final loaded or current configuration $\Omega$, see Fig. 6. Two intermediate configurations have been added to define the constitutive equations for the plastic mechanisms: slip plasticity and transformation plasticity. The first one is referred to as intermediate transformed configuration $\bar{\Omega}$ in which only the phase transformation part of the 
deformation gradient tensor $\mathbf{F}^{\mathrm{p}: \text { tr }}$ is accounted for. The second one is referred to as intermediate relaxed configuration $\overline{\bar{\Omega}}$ in which both phase transformation and slip shearing are considered. For an arbitrary material point $\mathbf{X}$ defined in the undeformed or reference configuration $\Omega_{0}$, the current configuration $\Omega$ is reached through the mapping $\mathbf{x}=\varphi(\mathbf{X}, t)$, where $\varphi$ is a mapping function and $t$ is the time. The deformation gradient $\mathbf{F}$, velocity $\mathbf{v}$ and velocity gradient $\mathbf{l}$ tensor are derived as:

$$
\left\{\begin{array}{l}
\mathbf{F}=\nabla_{\mathbf{X}} \varphi \\
\mathbf{v}=\dot{\varphi} \\
\mathbf{l}=\nabla_{x} \mathbf{v}=\dot{\mathbf{F}} \mathbf{F}^{-1}
\end{array}\right.
$$

The total deformation gradient tensor $\mathbf{F}$ is multiplicatively decomposed into an elastic part $\mathbf{F}^{\mathrm{e}}$, which includes the crystal lattice distortion and local rigid rotations, and a plastic part $\mathbf{F}^{\mathrm{p}}[42]$ :

$$
\mathbf{F}=\mathbf{F}^{\mathrm{e}} \mathbf{F}^{\mathrm{p}}=\mathbf{F}^{\mathrm{e}} \mathbf{F}^{\mathrm{p}: \mathrm{s}} \mathbf{F}^{\mathrm{p}: \operatorname{tr}}
$$

The plastic deformation gradient tensor $\mathbf{F}^{\mathrm{p}}$ is decomposed into the contributions of the phase transformation $\mathbf{F}^{\mathrm{p}: t r}$ and the slip shearing $\mathbf{F}^{\mathrm{p}: \mathrm{s}}$. The jacobian associated with the slip shearing retains the unimodal property $J^{\mathrm{p}: \mathrm{s}}=\operatorname{det}\left(\mathbf{F}^{\mathrm{p}: \mathrm{s}}\right)=1$, while a dilatational component $J^{\text {p:tr }}=\operatorname{det}\left(\mathbf{F}^{\text {p:tr }}\right) \neq 1$ can be expected in the case of the phase transformation.

The velocity gradient of the unified branch can be obtained by time-differentiating Eq. (3) as:

$$
\mathbf{l}=\dot{\mathbf{F}} \mathbf{F}^{-1}=\mathbf{l}^{\mathrm{e}}+\mathbf{F}^{\mathrm{e}} \overline{\overline{\mathbf{L}}}^{\mathrm{p}: \mathrm{s}} \mathbf{F}^{-\mathrm{e}}+\mathbf{F}^{\mathrm{e}} \overline{\overline{\mathbf{L}}}^{\mathrm{p}: \operatorname{tr}} \mathbf{F}^{-\mathrm{e}}
$$

where $\mathbf{l}^{\mathrm{e}}$ is the elastic contribution to the velocity gradient in the current configuration $\Omega, \overline{\overline{\mathbf{L}}}^{\mathrm{p}: \mathrm{s}}$ is the plastic component associated with standard slip and $\overline{\overline{\mathbf{L}}}^{\text {p:tr }}$ is the contribution of the irreversible phase transformation, both in the 

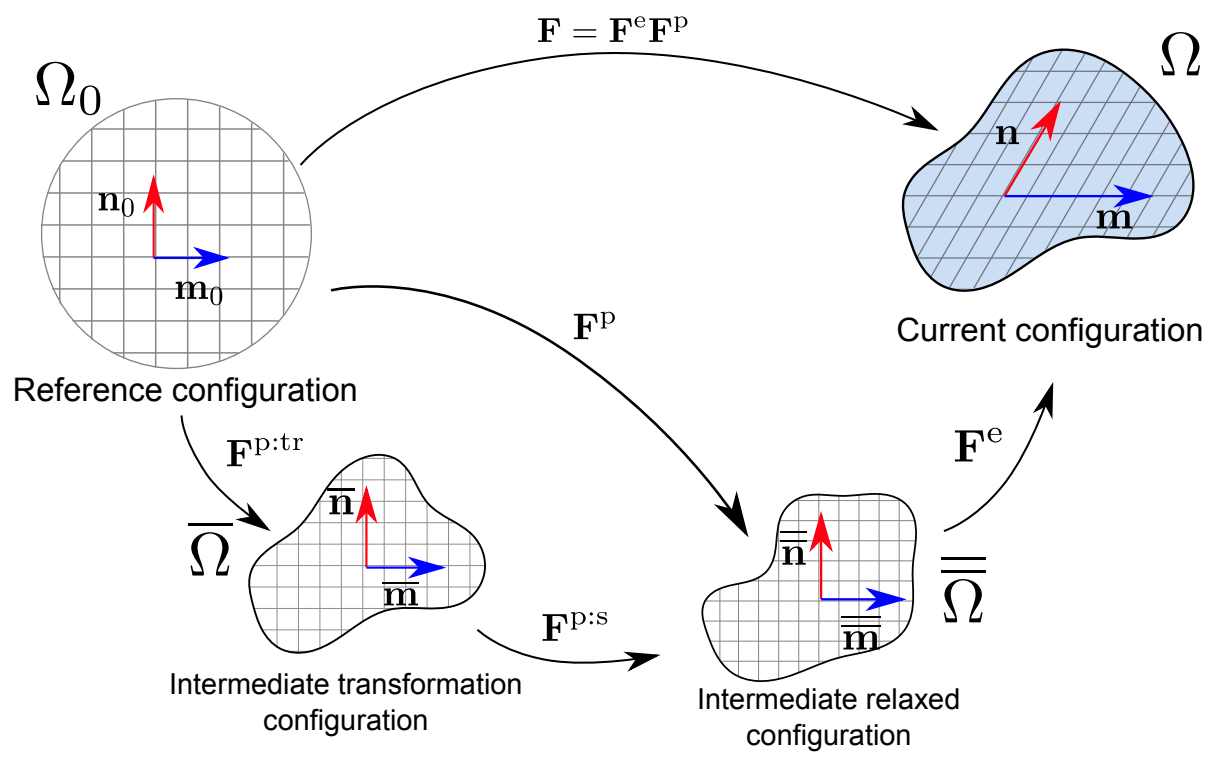

Figure 6: Kinematics of the proposed model showing the reference or initial configuration $\Omega_{0}$, the intermediate transformation configuration $\bar{\Omega}$, the intermediate relaxed configuration $\overline{\bar{\Omega}}$, and the current or loaded configuration $\Omega$. 
intermediate configuration $\overline{\bar{\Omega}}$ :

$$
\left\{\begin{array}{l}
\mathbf{l}^{\mathrm{e}}=\dot{\mathbf{F}}^{\mathrm{e}} \mathbf{F}^{-\mathrm{e}} \\
\overline{\overline{\mathbf{L}}}^{\mathrm{p}: \mathrm{s}}=\dot{\mathbf{F}}^{\mathrm{p}: \mathrm{s}} \mathbf{F}^{-\mathrm{p}: \mathrm{s}} \\
\overline{\overline{\mathbf{L}}}^{\mathrm{p}: \mathrm{tr}}=\mathbf{F}^{-\mathrm{p}: \mathrm{s}} \dot{\mathbf{F}}^{\mathrm{p}: \mathrm{tr}} \mathbf{F}^{-\mathrm{p}: \operatorname{tr}} \mathbf{F}^{-\mathrm{p}: \mathrm{s}}
\end{array}\right.
$$

These velocity gradients can be broken down into their symmetric $\left(\mathbf{d}^{\mathrm{e}}, \overline{\overline{\mathbf{D}}}^{\mathrm{p}: \mathrm{s}}\right.$ and $\overline{\overline{\mathbf{D}}}^{\text {p:tr }}$ ) and skew parts ( $\mathbf{w}^{\mathrm{e}}, \overline{\overline{\mathbf{W}}}^{\text {p:s }}$ and $\left.\overline{\overline{\mathbf{W}}}^{\text {p:tr }}\right)$ :

$$
\left\{\begin{array}{l}
\mathbf{l}^{\mathrm{e}}=\mathbf{d}^{\mathrm{e}}+\mathbf{w}^{\mathrm{e}} \\
\overline{\overline{\mathbf{L}}}^{\mathrm{p}: \mathrm{s}}=\overline{\overline{\mathbf{D}}}^{\mathrm{p}: \mathrm{s}}+\overline{\overline{\mathbf{W}}}^{\mathrm{p}: \mathrm{s}} \\
\overline{\overline{\mathbf{L}}}^{\mathrm{p}: \operatorname{tr}}=\overline{\overline{\mathbf{D}}}^{\mathrm{p}: \operatorname{tr}}+\overline{\overline{\mathbf{W}}}^{\mathrm{p}: \mathrm{tr}}
\end{array}\right.
$$

expressed in $\Omega_{0}$ for the elastic terms and in $\overline{\bar{\Omega}}$ for the plastic terms.

\subsection{Thermodynamics}

In this section, the first and second Thermodynamics Principles are taken as the starting point in order to derive the thermomechanical forces for the different deformation modes (elastic, slip and phase transformation), the elastic response of each phase and to verify the thermodynamical consistency of the constitutive model developed herein. To this end, the Thermodynamic Principles and the free energy function $\psi_{0}$ are expressed in the reference configuration $\Omega_{0}$, since the evolution of the transformed phase is tracked in this configuration. However, the plastic terms are expressed in the dilated configuration $\overline{\bar{\Omega}}$ where the flow rules are usually defined [43]. The local form of the energy balance in the reference configuration, assuming constant and uniform temperature $T$ within the body and neglecting heat terms, can be expressed as (see Appendix A for detailed 
expressions):

$$
\dot{e}_{0}=J^{\mathrm{p}}\left(\overline{\overline{\mathbf{M}}}: \overline{\overline{\mathbf{L}}}^{\mathrm{p}: \mathrm{s}}+\overline{\overline{\mathbf{M}}}_{0}: \overline{\overline{\mathbf{L}}}^{\mathrm{p}: \mathrm{tr}}\right)+\mathbf{S}_{0}: \mathbf{F}^{T} \mathbf{d}^{\mathrm{e}} \mathbf{F}
$$

where $\dot{e}_{0}$ is the rate of specific internal energy $e_{0}$ per unit reference volume, $\mathbf{S}_{0}=J \mathbf{F}^{-1} \boldsymbol{\sigma} \mathbf{F}^{-T}$ is the second Piola-Kirchhoff stress of the homogenized system resistance (parent + transformed phase) in the reference configuration, and $\overline{\overline{\mathbf{M}}}=\mathbf{F}^{\mathrm{e} T} \mathbf{F}^{\mathrm{e}} \overline{\overline{\mathbf{S}}}$ is the Mandel stress tensor in $\overline{\bar{\Omega}}$, with $\overline{\overline{\mathbf{S}}}=J^{\mathrm{e}} \mathbf{F}^{\mathrm{e}-1} \boldsymbol{\sigma} \mathbf{F}^{\mathrm{e}-T}$ being the second Piola-Kirchhoff stress in $\overline{\bar{\Omega}}$.

The second principle in the reference configuration can be simplified in its local form to (see Appendix A for more details):

$$
\dot{\eta}_{0} \geq 0
$$

where $\dot{\eta}_{0}$ is the rate of specific internal entropy $\eta_{0}$ per reference unit volume.

The specific internal entropy $\eta_{0}$ and the specific internal energy $e_{0}$ are both linked by the definition of the Helmholtz specific free energy $\psi_{0}=e_{0}-T \eta_{0}$, where $T$ is the absolute temperature.

The combination of the first and the second Thermodynamics Principles (Eqs. (7) and (8)) along with the free energy rate $\dot{\psi}_{0}=\dot{e}_{0}-\dot{T} \eta_{0}-T \dot{\eta}_{0}$ leads to the following expression of the Clausius-Duhem inequality (assuming constant temperature):

$$
-\dot{\psi}_{0}+J^{\mathrm{p}}\left(\overline{\overline{\mathbf{M}}}: \overline{\overline{\mathbf{L}}}^{\mathrm{p}: \mathrm{s}}+\overline{\overline{\mathbf{M}}}: \overline{\overline{\mathbf{L}}}^{\mathrm{p}: \mathrm{tr}}\right)+\mathbf{S}_{0}: \mathbf{F} \mathbf{d}^{\mathrm{e}} \mathbf{F} \geq 0
$$

where the left-hand side term represents the dissipation rate per unit reference volume.

The Helmholtz free energy function of the homogenized material $\psi_{0}$ is as- 
sumed to be the combination of the contribution associated with the parent domain $\psi_{0}^{\mathrm{pa}}$ and the transformed domain $\psi_{0}^{\mathrm{tr}}$ :

$$
\psi_{0}=\left(1-f_{0}\right) \psi_{0}^{\mathrm{pa}}+f_{0} \psi_{0}^{\mathrm{tr}}
$$

where $f_{0}$ is the volume fraction of material that has undergone the phase transformation in the reference configuration $\Omega_{0}$ and corresponds to the joint addition of the contributions of all the phase transformation systems or twin systems $f_{0}=\sum_{\beta} f_{0: \beta}$. The free energy function for both phases is assumed to be a function of the elastic right Cauchy-Green tensor $\mathbf{C}^{\mathrm{e}}=\mathbf{F}^{e T} \mathbf{F}^{\mathrm{e}}$ and the mass concentration of the studied elements (Co and $\mathrm{Cr}$ ) in each phase, $c_{\mathrm{pa}}$ and $c_{\mathrm{tr}}$. These are defined as mass of solute per total mass $\left(c_{\mathrm{sol}}=\frac{m_{\mathrm{sol}}}{m_{\mathrm{tot}}}\right)$. The total mass of these solute elements within both phases reads:

$$
m_{\mathrm{Co}+\mathrm{Cr}}=V_{0}\left(\left(1-f_{0}\right) \rho_{0: \mathrm{pa}} c_{\mathrm{pa}}+f_{0} \rho_{0: \mathrm{tr}} c_{\mathrm{tr}}\right)
$$

Supported by experimental observations [4, 9, 3], the concentration differences are small enough ( $1 \%$ to $2 \%$ ) to assume that the density of both phases is the same in the reference configuration $\left(\rho_{0: \mathrm{pa}}=\rho_{0: \mathrm{tr}}\right)$. Taking this into account, the time derivative of Eq. 111 and assuming mass conservation of the diffusive species $\left(\dot{m}_{\mathrm{Co}+\mathrm{Cr}}=0\right)$, one reaches:

$$
\left(1-f_{0}\right) \dot{c}_{\mathrm{pa}}=-f_{0} \dot{c}_{\mathrm{tr}}-\dot{f}_{0}\left(c_{\mathrm{tr}}-c_{\mathrm{pa}}\right)
$$

The time derivative of $\psi_{0}$ defined in Eq. (10) then reads

$$
\begin{aligned}
\dot{\psi}_{0}=\left[\left(1-f_{0}\right) \frac{\partial \psi_{0}^{\mathrm{pa}}}{\partial \mathbf{C}^{\mathrm{e}}}+\right. & \left.f_{0} \frac{\partial \psi_{0}^{\mathrm{tr}}}{\partial \mathbf{C}^{\mathrm{e}}}\right]: \dot{\mathbf{C}}^{\mathrm{e}}-\psi_{0}^{\mathrm{pa}} \dot{f}_{0}+\psi_{0}^{\mathrm{tr}} \dot{f}_{0} \\
& +f_{0} \frac{\partial \psi_{0}^{\mathrm{tr}}}{\partial c_{\mathrm{tr}}} \dot{c}_{\mathrm{tr}}-f_{0} \frac{\partial \psi_{0}^{\mathrm{pa}}}{\partial c_{\mathrm{pa}}} \dot{c}_{\mathrm{tr}}-\dot{f}_{0}\left(c_{\mathrm{tr}}-c_{\mathrm{pa}}\right) \frac{\partial \psi_{0}^{\mathrm{pa}}}{\partial c_{\mathrm{pa}}}
\end{aligned}
$$


Substituting the definition of the right Cauchy-Green deformation tensor $\mathbf{C}^{\mathrm{e}}$ and rearranging, the term $\frac{\partial \psi_{0}^{i}}{\partial \mathbf{C}^{\mathrm{e}}}: \dot{\mathbf{C}}^{\mathrm{e}}$ (where $i$ stands for the parent phase or the transformed phase) can be rewritten as:

$$
\frac{\partial \psi_{0}^{i}}{\partial \mathbf{C}^{\mathrm{e}}}: \dot{\mathbf{C}}^{\mathrm{e}}=2 \frac{\partial \psi_{0}^{i}}{\partial \mathbf{C}^{\mathrm{e}}}: \mathbf{F}^{e T} \mathbf{d}^{\mathrm{e}} \mathbf{F}^{\mathrm{e}}
$$

Introducing this in Eq. 13 and substituting this expression for $\dot{\psi}_{0}$ into Eq. (9), the Clausius-Duhem inequality reads

$$
\begin{aligned}
& \underbrace{\left(\mathbf{F}^{\mathrm{p}} \mathbf{S}_{0} \mathbf{F}^{\mathrm{p} T}-2\left[\left(1-f_{0}\right) \frac{\partial \psi_{0}^{\mathrm{pa}}}{\partial \mathbf{C}^{\mathrm{e}}}+f_{0} \frac{\partial \psi_{0}^{\mathrm{tr}}}{\partial \mathbf{C}^{\mathrm{e}}}\right]\right): \mathbf{F}^{\mathrm{e} T} \mathbf{d}^{\mathrm{e}} \mathbf{F}^{\mathrm{e}}}_{\text {elastic }}+\underbrace{J^{\mathrm{p}}(\overline{\overline{\mathbf{M}}}: \overline{\overline{\mathbf{L}}} \mathrm{p}: \mathrm{s})}_{\text {slip }} \\
& \underbrace{J^{\mathrm{p}}\left(\overline{\overline{\mathbf{M}}}: \overline{\overline{\mathbf{L}}}^{\mathrm{p}: \mathrm{tr}}\right)+\left(\psi_{0}^{\mathrm{pa}}-\psi_{0}^{\mathrm{tr}}+\left(c_{\mathrm{tr}}-c_{\mathrm{pa}}\right) \frac{\partial \psi_{0}^{\mathrm{pa}}}{\partial c_{\mathrm{pa}}}\right) \dot{f}_{0}}_{\text {transformation }}+\underbrace{f_{0}\left(\frac{\partial \psi_{0}^{\mathrm{pa}}}{\partial c_{\mathrm{pa}}}-\frac{\partial \psi_{0}^{\mathrm{tr}}}{\partial c_{\mathrm{tr}}}\right) \dot{c}_{\mathrm{tr}}}_{\text {diffusion }} \geq 0
\end{aligned}
$$

Here, the transformation term $\left(c_{\mathrm{tr}}-c_{\mathrm{pa}}\right) \frac{\partial \psi_{0}^{\mathrm{pa}}}{\partial c_{\mathrm{pa}}}$ is small compared to the other high energy transformation terms if the parent phase does not contain any high energy defect or is near equilibrium $\left(\left(c_{\mathrm{tr}}-c_{\mathrm{pa}}\right) \frac{\partial \psi_{0}^{\mathrm{pa}}}{\partial c_{\mathrm{pa}}} \ll \psi_{0}^{\mathrm{pa}}-\psi_{0}^{\mathrm{tr}}\right)$. This assumption is valid for the present work and, therefore, the transformation term can be omitted:

$$
\begin{array}{r}
\underbrace{\left(\mathbf{F}^{\mathrm{p}} \mathbf{S}_{0} \mathbf{F}^{\mathrm{p} T}-2\left[\left(1-f_{0}\right) \frac{\partial \psi_{0}^{\mathrm{pa}}}{\partial \mathbf{C}^{\mathrm{e}}}+f_{0} \frac{\partial \psi_{0}^{\mathrm{tr}}}{\partial \mathbf{C}^{\mathrm{e}}}\right]\right): \mathbf{F}^{\mathrm{e} T} \mathbf{d}^{\mathrm{e}} \mathbf{F}^{\mathrm{e}}}_{\text {elastic }}+\underbrace{J^{\mathrm{p}}\left(\overline{\overline{\mathbf{M}}}: \overline{\left.\overline{\mathbf{L}}^{\mathrm{p}: \mathrm{s}}\right)}\right.}_{\text {slip }} \\
+\underbrace{J^{\mathrm{p}}\left(\overline{\overline{\mathbf{M}}}: \overline{\overline{\mathbf{L}}}^{\mathrm{p}: \operatorname{tr}}\right)+\left(\psi_{0}^{\mathrm{pa}}-\psi_{0}^{\mathrm{tr}}\right) \dot{f}_{0}}_{\text {transformation }}+\underbrace{f_{0}\left(\frac{\partial \psi_{0}^{\mathrm{pa}}}{\partial c_{\mathrm{pa}}}-\frac{\partial \psi_{0}^{\mathrm{tr}}}{\partial c_{\mathrm{tr}}}\right) \dot{c}_{\mathrm{tr}}}_{\text {diffusion }} \geq 0
\end{array}
$$

The Coleman and Noll method 44 establishes that the fundamental inequality presented in Eq. (16) must be satisfied for every admissible thermodynamic 
process. Accordingly, the elastic term must be equal to zero for any $\mathbf{F}^{\mathrm{e} T} \mathbf{d}^{\mathrm{e}} \mathbf{F}^{\mathrm{e}}$, leading to the constitutive equation:

$$
\overline{\overline{\mathbf{S}}}=2 J^{p-1}\left[\left(1-f_{0}\right) \frac{\partial \psi_{0}^{\mathrm{pa}}}{\partial \mathbf{C}^{\mathrm{e}}}+f_{0} \frac{\partial \psi_{0}^{\mathrm{tr}}}{\partial \mathbf{C}^{\mathrm{e}}}\right]
$$

Introducing this expression into Eq. (16) reduces the Clausius-Duhem inequality to:

$$
\underbrace{J^{\mathrm{p}}\left(\overline{\overline{\mathbf{M}}}: \overline{\overline{\mathbf{L}}}^{\mathrm{p}: \mathrm{s}}\right)}_{\text {slip }}+\underbrace{J^{\mathrm{p}}\left(\overline{\overline{\mathbf{M}}}: \overline{\overline{\mathbf{L}}}^{\mathrm{p}: \mathrm{tr}}\right)+\left(\psi_{0}^{\mathrm{pa}}-\psi_{0}^{\mathrm{tr}}\right) \dot{f}_{0}}_{\text {transformation }}+\underbrace{f_{0}\left(\frac{\partial \psi_{0}^{\mathrm{pa}}}{\partial c_{\mathrm{pa}}}-\frac{\partial \psi_{0}^{\mathrm{tr}}}{\partial c_{\mathrm{tr}}}\right) \dot{c}_{\mathrm{tr}}}_{\text {diffusion }} \geq 0
$$

where, as stated by the second Thermodynamics Principle, the left-hand side of this equation represents the rate of internal entropy production $\dot{\eta}_{0}$ and is equal to the dissipation rate per unit reference volume $\dot{\mathcal{D}}$. The first term in Eq. (18) is the dissipation associated with the plastic deformation due to slip, the second term comes from the dissipation produced by the plastic transformation and the third term is the dissipation associated to the chemical change of the body driven by the diffusion between phases. The thermodynamic consistency of the constitutive framework developed herein is verified in Appendix B.

\subsection{Constitutive model}

\subsubsection{Elastic behavior}

The Helmholtz free energy of each phase $i$ is assumed to be additively decomposed into a mechanical term $\psi_{0: \text { mech }}^{i}\left(\mathbf{E}^{\mathrm{e}}\right)$ dependent only on the elastic strain $\mathbf{E}^{\mathrm{e}}$ and a chemical term $\psi_{0 \text { :chem }}\left(c_{i}\right)$ dependent on the concentration of $\mathrm{Co}+\mathrm{Cr}$ in each phase:

$$
\psi_{0}^{i}=\psi_{0: \text { mech }}^{i}\left(\mathbf{E}^{\mathrm{e}}\right)+\psi_{0: \mathrm{chem}}^{i}\left(c_{i}\right)
$$


Deformation in metals for the conditions considered in this work usually leads to infinitesimal elastic strains and the mechanical part of the elastic strain energy can be defined as a quadratic function of the elastic strain:

$$
\psi_{0: \text { mech }}^{i}=\frac{1}{2} \mathbb{C}_{i}::\left(\mathbf{E}^{\mathrm{e}} \otimes \mathbf{E}^{\mathrm{e}}\right)
$$

where $\mathbb{C}_{i}$ represents the fourth-order elasticity tensor of phase $i$ and $\mathbf{E}^{\mathrm{e}}=\frac{1}{2}\left(\mathbf{C}^{e}-\right.$ I) is the Green-Lagrange elastic strain. Using this expression in Eq. 17), the second Piola-Kirchhoff stress tensor reads:

$$
\overline{\overline{\mathbf{S}}}=J^{p-1}\left[\left(1-f_{0}\right) \mathbb{C}_{\mathrm{pa}}+f_{0} \mathbb{C}_{\mathrm{tr}}\right]: \mathbf{E}^{\mathrm{e}}
$$

The fourth-order matrix elasticity tensor $\mathbb{C}_{i}$ can be formulated in crystal principal axes as:

$$
\mathbb{C}_{\mathrm{pa}}=\mathbf{R}_{\text {crys } \rightarrow \Omega_{0}} \mathbf{R}_{\text {crys } \rightarrow \Omega_{0}} \mathbb{C}_{\text {crys }} \mathbf{R}_{\text {crys } \rightarrow \Omega_{0}}^{T} \mathbf{R}_{\text {crys } \rightarrow \Omega_{0}}^{T}
$$

where $\mathbb{C}_{\text {crys }}$ is the stiffness matrix in the principal crystal axes and $\mathbf{R}_{\text {crys } \rightarrow \Omega_{0}}$ is the rotation matrix between the principal crystal configuration and the reference configuration. $\mathbb{C}_{\text {tr }}$ is related to $\mathbb{C}_{\text {crys }}$ by:

$$
\mathbb{C}_{\text {tr }}=\mathbf{R}_{\text {crys } \rightarrow \Omega_{0}} \mathbf{R}_{\text {crys } \rightarrow \Omega_{0}} \mathbf{R}_{\text {twin }} \mathbf{R}_{\text {twin }} \mathbb{C}_{\text {crys }} \mathbf{R}_{\text {twin }}^{T} \mathbf{R}_{\text {twin }}^{T} \mathbf{R}_{\text {crys } \rightarrow \Omega_{0}}^{T} \mathbf{R}_{\text {crys } \rightarrow \Omega_{0}}^{T}
$$

where $\mathbf{R}_{\text {twin }}$ represents the transformation matrix between the lattice orientation in the matrix and the lattice orientation in the twinned region. The rotation matrix $\mathbf{R}_{\text {twin }}$ is defined for the case of compound type twins in FCC by 45 ]

$$
\mathbf{R}_{\text {twin }}=2\left(\overline{\overline{\mathbf{m}}}_{\beta} \otimes \overline{\overline{\mathbf{m}}}_{\beta}\right)-\mathbf{I}
$$


where $\overline{\overline{\mathbf{m}}}_{\beta}$ represents the glide direction of the twin system $\beta$ in the relaxed configuration $\overline{\bar{\Omega}}$.

The free energy for stacking faults in metals is usually expressed in terms of the stacking fault energy $\Gamma_{\mathrm{SF}}$ defined as [46]:

$$
\Gamma_{\mathrm{SF}}=\frac{\partial \Psi}{\partial A_{\mathrm{f}}}=h\left(\psi_{0: \text { chem }}^{\mathrm{tr}}-\psi_{0: \text { chem }}^{\mathrm{pa}}\right)
$$

where $\Psi$ is the extensive free energy, $A_{\mathrm{f}}$ is the area of fault and $h$ the height of an individual fault or twin ledge.

Finally, the stacking fault energy $\Gamma_{\mathrm{SF}}$ of the new phase is defined as a function of its growth rate. For this purpose, the model presented by Barba et al. 44 is used in the present work. A summary of the model is presented in Appendix C. In this model, the growth velocity of the fault ledge is obtained by solving the diffusion problem in its surrounding and imposing the concentration at the fault. The energy of the growing fault profile as a function of the $\mathrm{Co}+\mathrm{Cr}$ concentration $c$ is presented in Fig. $7 \mathrm{~b}$. Here, three different scenarios can be identified, see Fig. 7 a. First, when the strain rates are high compared to the characteristic diffusion rate of the problem, the chemical problem can be considered as static and, therefore, the new phase is growing with the same concentration as the parent phase. This implies that the fault is growing unsegregated with a high stacking fault energy corresponding to a complex stacking fault $\left(\Gamma_{\mathrm{SF}}=\Gamma_{2 \mathrm{xCISF}}=182 \mathrm{~mJ} / \mathrm{mm}^{2}\right.$ 34] $)$. This is indicated as scenario A in Fig. $7 \mathrm{a}$-b. The concentration threshold for scenario $\mathrm{A}$ is considered to be the fault concentration experimentally found for MD2 by Barba et al. [4]. On the other hand, if the phase transformation occurs slowly enough, the fault can be considered to be in fully segregated equilibrium, therefore, with a low energy corresponding to a standard $\operatorname{SESF}\left(\Gamma_{\mathrm{SF}}=74 \mathrm{~mJ} / \mathrm{mm}^{2}\right.$ [34] $)$. This is indicated as scenario C in Fig. 7a-b. Between these two extreme cases, the growing fault 
is partially in equilibrium and the staking fault energy is assumed to follow a linear relation with the $\mathrm{Co}+\mathrm{Cr}$ concentration of the fault as indicated in scenario B [34. Bringing together both functions in Fig. $7 \mathrm{a}$-b leads to the definition of the new phase staking fault energy depending on the fault lengthening rate, see Fig. 77.

\subsubsection{Plastic relations}

\subsubsection{Plastic slip flow}

From the kinematics of dislocation motion, the plastic velocity gradient $\overline{\overline{\mathbf{L}}}^{\text {:s }}$ is of the form [47]:

$$
\overline{\overline{\mathbf{L}}}^{\mathrm{p}: \mathrm{s}}=\sum_{\alpha} \dot{\gamma}_{\alpha} \overline{\overline{\mathbf{m}}}_{\alpha} \otimes \overline{\overline{\mathbf{n}}}_{\alpha}
$$

where $\dot{\gamma}_{\alpha}$ is the shear rate of the slip system $\alpha$ and $\overline{\overline{\mathbf{n}}}_{\alpha}$ is the corresponding slip

plane normal defined in the relaxed configuration $\overline{\bar{\Omega}}\left(\overline{\overline{\mathbf{m}}}_{\alpha}\right.$ is the same as in Eq. (24)).

The plastic flow described in (26) is defined by the plastic multiplier $\dot{\gamma}_{\alpha}$. The evolution of this multiplier is governed by the conjugate thermodynamic forces for each mechanism which can be identified from the expression of the mechanical dissipation sources in Eq. (18). The driving force for slip shearing per unit of volume in the intermediate configuration $\overline{\bar{\Omega}}$ can be rewritten as:

$$
\dot{\overline{\mathcal{D}}}_{\text {slip }}=\left(\overline{\overline{\mathbf{M}}}: \overline{\overline{\mathbf{L}}}^{\mathrm{p}: \mathrm{s}}\right)=\sum_{\alpha} \overline{\overline{\mathbf{M}}}:\left(\overline{\overline{\mathbf{m}}}_{\alpha} \otimes \overline{\overline{\mathbf{n}}}_{\alpha}\right) \dot{\gamma}_{\alpha}=\sum_{\alpha} \tau_{\alpha} \dot{\gamma}_{\alpha}
$$

where $\tau_{\alpha}=\overline{\overline{\mathbf{M}}}:\left(\overline{\overline{\mathbf{m}}}_{\alpha} \otimes \overline{\overline{\mathbf{n}}}_{\alpha}\right)$ is the slip resolved shear stress on slip system $\alpha$ and corresponds to the driving force for slip shearing.

The evolution of the plastic multiplier $\dot{\gamma}_{\alpha}$ can be described by viscous flow rules dependent on the conjugate thermodynamic force $\tau_{\alpha}$. While more complex relationships exist, e.g. [48, they can be linked through a simple power-law 
a) Fault structure

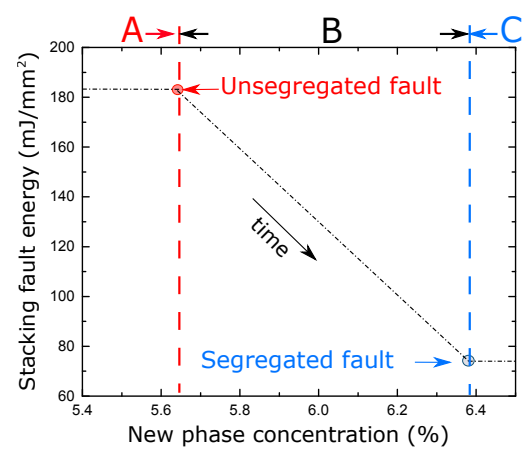

b) Diffusion

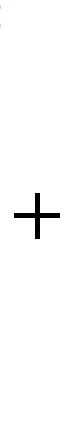

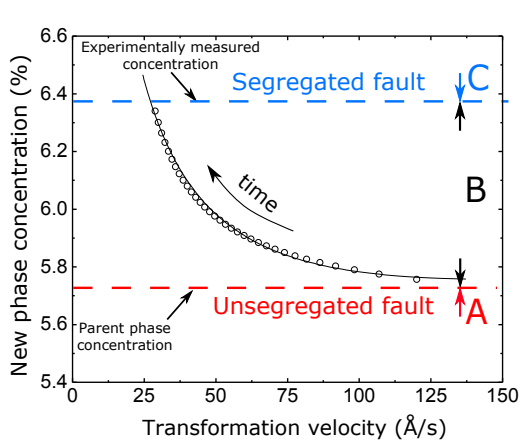

c) Staking fault energy $\Gamma_{\mathrm{SF}}$

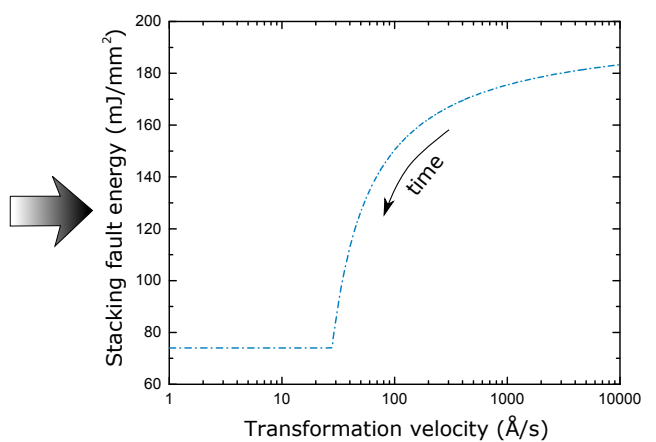

Figure 7: a) Stacking fault energy $\Gamma_{\mathrm{SF}}$ as a function of the concentration of the new phase. Energies for the perfect CSF (unsegregated fault) and SESF (segregated fault) are obtained from Voronstov et al. 34]; b) fault concentration of the new phase $c_{\operatorname{tr}}$ as a function of the transformation velocity $v_{\mathrm{tr}}$ obtained from the diffusion model of Barba et al. 4, points are the exact solution for the diffusion problem; c) the combination of the functions presented in a) and b) leads to the stacking fault energy for the phase transformation problem vs. its fault growth rate. 
[49, 50]:

$$
\dot{\gamma}_{\alpha}=\dot{\gamma}_{0}\left(\frac{\tau_{\alpha}}{s_{\alpha}}\right)^{1 / m_{\alpha}}
$$

where $m_{\alpha}=\frac{Q_{\text {act }}}{k_{B} T}$, with $k_{B}$ as the Boltzmann constant, $T$ is the absolute temperature and $Q_{\text {act }}$ is the activation energy of the deformation mechanism. Non-explicit yielding is considered in this model. If $\tau_{\alpha} \neq 0$, the material is always plastically deforming along the slip plane following the positive direction of $\tau_{\alpha}$. This approach has been used successfully before in other creep models [51, 52, 53].

\subsubsection{Phase transformation flow}

Extending the same approach to the phase transformation, $\mathbf{F}^{\mathrm{p}: t r}$ accounts for two contributions associated with each phase transformation system $\beta$ :

- Shear strain $\gamma_{\text {tr }}$ that follows a slip direction $\overline{\mathbf{m}}_{\beta}$ and is associated with the Schmid tensor $\overline{\overline{\mathbf{m}}}_{\beta} \otimes \overline{\overline{\mathbf{n}}}_{\beta}$, where $\overline{\overline{\mathbf{n}}}_{\beta}$ is the corresponding transformation plane normal.

- Volumetric strain $\delta_{\mathrm{tr}: \beta}=J^{\mathrm{tr}}-1$ where $J^{\mathrm{tr}}=\operatorname{det} \mathbf{F}^{\mathrm{tr}}$ : this transformation can be expressed as a function of a unit-modulus tensor $\mathbf{F}_{\Delta V}$ that determines the volumetric part of the phase transformation gradient and is escalated by the volumetric multiplier $\delta_{\mathrm{tr}: \beta}$.

The transformation velocity gradient then reads [54]:

$$
\overline{\overline{\mathbf{L}}}^{\mathrm{p}: \operatorname{tr}}=\sum_{\beta} \dot{f}_{0: \beta}\left[\left(\gamma_{\mathrm{tr}}\left(\overline{\overline{\mathbf{m}}}_{\beta} \otimes \overline{\overline{\mathbf{n}}}_{\beta}\right)+\delta_{\mathrm{tr}: \beta} \mathbf{F}_{\Delta V}\right]\right.
$$

where $\dot{f}_{0: \beta}$ is the fraction rate of transformed phase $\beta$.

For the case of microtwins and stacking faults in superalloys, where the transformation consists of a simple shear, the dilatational term is negligible $\left(\delta_{\mathrm{tr}: \beta}=J^{\mathrm{tr}}-1=J^{\mathrm{p}}-1=0\right)$ and, therefore, $\dot{f}_{\beta}=\dot{f}_{0: \beta}$ is assumed from this 
point onwards [9, 6]. Thus, the velocity gradient $\overline{\overline{\mathbf{L}}}^{\text {:tr }}$ reduces to:

$$
\overline{\overline{\mathbf{L}}}^{\mathrm{p}: \operatorname{tr}}=\sum_{\beta} \dot{\gamma}_{\beta}\left(\overline{\overline{\mathbf{m}}}_{\beta} \otimes \overline{\overline{\mathbf{n}}}_{\beta}\right)
$$

where the plastic multiplier $\dot{\gamma}_{\beta}$ is related to the transformation fraction rate $\dot{f}_{0: \beta}$ by:

$$
\dot{\gamma}_{\beta}=\dot{f}_{0: \beta} \gamma_{\mathrm{tr}}
$$

Similarly to slip, the driving force for phase transformation can be extracted from the dissipation rate:

$$
\begin{aligned}
& \dot{\overline{\mathcal{D}}}_{\text {transformation }}=\overline{\overline{\mathbf{M}}}: \overline{\overline{\mathbf{L}}}^{\mathrm{p}: \operatorname{tr}}+J^{-\mathrm{p}}\left(\psi_{0}^{\mathrm{pa}}-\psi_{0}^{\mathrm{tr}}\right) \dot{f}_{0}= \\
& \sum_{\beta}\left[\overline{\overline{\mathbf{M}}}:\left(\overline{\overline{\mathbf{m}}}_{\beta} \otimes \overline{\overline{\mathbf{n}}}_{\beta}\right) \gamma_{\mathrm{tr}}+J^{-\mathrm{p}}\left(\psi_{0}^{\mathrm{pa}}-\psi_{0}^{\mathrm{tr}}\right)\right] \dot{f}_{0: \beta}=\sum_{\beta}\left[\tau_{\beta}+J^{-\mathrm{p}} \frac{\psi_{0}^{\mathrm{pa}}-\psi_{0}^{\mathrm{tr}}}{\gamma_{\mathrm{tr}: \beta}}\right] \dot{\gamma}_{\beta}
\end{aligned}
$$

where $\tau_{\beta}=\overline{\overline{\mathbf{M}}}:\left(\overline{\overline{\mathbf{m}}}_{\beta} \otimes \overline{\overline{\mathbf{n}}}_{\beta}\right)$ is the phase transformation resolved shear stress of the phase transformation system $\beta$. The driving force for phase transformation assuming plastic $\left(J^{\mathrm{p}}=1\right)$ isochoricity is then defined as $\tau_{\beta}+\frac{\psi_{0}^{\mathrm{pa}}-\psi_{0}^{\mathrm{tr}}}{\gamma_{\mathrm{tr}}}$. Making use of the free energy split into elastic $\psi_{0 \text { :mech }}$ and chemical contributions $\psi_{0: c h e m}$, we can express this driving force in terms of the elastic strain $\mathbf{E}^{\mathrm{e}}$ and the stacking fault energy $\Gamma_{\mathrm{SF}}$ from Eqs. 250,219$)$ and $(20)$ as:

$$
\tau_{\beta}+\frac{\psi_{0}^{\mathrm{pa}}-\psi_{0}^{\mathrm{tr}}}{\gamma_{\mathrm{tr}}}=\tau_{\beta}-\frac{\Gamma_{\mathrm{SF}: \beta}}{\gamma_{\mathrm{tr}} h}-\frac{1}{2 \gamma_{\mathrm{tr}}}\left(\mathbb{C}_{\mathrm{tr}}-\mathbb{C}_{\mathrm{pa}}\right)::\left(\mathbf{E}^{\mathrm{e}} \otimes \mathbf{E}^{\mathrm{e}}\right)
$$

Following the procedure introduced by Mareau et al. [50 for magnesium and Meric et al. 49] for FCC materials, this driving force can be introduced in the numerator term of the power law in Eq. 28). The flow rule for the plastic 
multiplier $\dot{\gamma}_{\beta}$ follows

$$
\begin{cases}\dot{\gamma}_{\beta}=\dot{\gamma}_{0}\left(\frac{\tau_{\beta}-\frac{\Gamma_{\mathrm{SF}: \beta}}{\gamma_{\mathrm{tr}} h}-\frac{1}{2 \gamma_{\mathrm{tr}}}\left(\mathbb{C}_{\mathrm{tr}}-\mathbb{C}_{\mathrm{pa}}\right)::\left(\mathbf{E}^{\mathrm{e}} \otimes \mathbf{E}^{\mathrm{e}}\right)}{s_{\beta}}\right)^{1 / m_{\beta}}, & \text { if } \tau_{\beta}-\frac{\Gamma_{\mathrm{SF}: \beta}}{\gamma_{\mathrm{tr}} h}-\frac{1}{2 \gamma_{\mathrm{tr}}}\left(\mathbb{C}_{\mathrm{tr}}-\mathbb{C}_{\mathrm{pa}}\right)::\left(\mathbf{E}^{\mathrm{e}} \otimes \mathbf{E}^{\mathrm{e}}\right)>0 \\ \dot{\gamma}_{\beta}=0, & \text { if } \tau_{\beta}-\frac{\Gamma_{\mathrm{SF}: \beta}}{\gamma_{\mathrm{tr}} h}-\frac{1}{2 \gamma_{\mathrm{tr}}}\left(\mathbb{C}_{\mathrm{tr}}-\mathbb{C}_{\mathrm{pa}}\right)::\left(\mathbf{E}^{\mathrm{e}} \otimes \mathbf{E}^{\mathrm{e}}\right) \leq 0\end{cases}
$$

where $m_{\beta}$ is the rate dependency coefficient defined as in the slip case. The plastic flow rule associated with the phase transformation is assumed to be unidirectional. Indeed, as detwinning is unlikely to happen under uniaxial loading conditions, it is not considered in this work, as done elsewhere [55, 56].

\subsubsection{Hardening laws}

The strain hardening of the viscous resistance parameters $\dot{s}_{i}$ is defined by the generalized rate equation:

$$
\dot{s}_{\beta}=\sum_{j} h_{i j} \dot{\gamma}_{j}
$$

where $h_{i j}$ are the hardening moduli. The sum ranges over all the deformation mechanisms (slip+twin). The hardening moduli evolution is defined as [57]:

$$
h_{i j}=\left(q+(1-q) \delta_{i j}\right) h_{0} \operatorname{sech}^{2}\left(\frac{h_{0} \gamma_{i}}{s_{s}-s_{0}}\right)\left(1+\sum_{i \neq j} f_{i j} \tanh \left(\gamma_{j} / \gamma_{0}\right)\right)
$$

where $q$ is the cross hardening parameter, $\delta_{i j}$ is the Kronecker symbol, $h_{0}$ is the hardening modulus, $s_{0}$ is the initial shear resistance, $s_{s}$ is the breakthrough stress at which the large plastic flow initiates, $\gamma_{0}$ is the amount of shear strain after which the interaction between the shear systems reaches a peak strength and $f_{i j}$ represents the degree of interaction between deformation mechanisms. For the sake of simplicity, only the strong hardening interaction of the twin bands on the other non-coplanar twin/slip systems is considered $\left(f_{i j} \neq 0\right.$ if $j \in$ 
twin systems and $i$ and $j$ are not coplanar). This is believed to be the strongest hardening mechanism because of its high effectiveness in reducing the free path for dislocations. Hardening between the coplanar slip or twin systems is not considered.

As a summary, Fig. 8 presents the proposed multiscale approach for the phase transformation problem. First, the viscous growth rate for the phase transformation $\dot{f}_{0}$ is defined in terms of the free energy function $\psi_{\mathrm{tr}}-\psi_{\mathrm{pa}}$ using the Thermodynamics Principles. Then, the free energy is linked with the concentration of the new phase $c_{\mathrm{tr}}$ using atomistic data from the literature [34. Linear evolution of the fault energy with respect to the concentration is assumed. Finally, the concentration of the new phase is defined as a function of its growth rate using diffusion theory [4]. The set of nonlinear diffusion equations are coupled with the mechanics of the constitutive problem and thus need to be solved simultaneously. The set of diffusion equations linking the fault concentration and its growth rate are detailed in Eqs. (C4) in Appendix C. This set of nonlinear diffusion equations is coupled to the mechanics of the constitutive problem through the strain rate - stacking fault energy relationship in Eq. (34) and thus need to be solved simultaneously.

\section{Simulations of creep in single crystal MD2 Ni-based superalloy}

The continuum model described above was implemented in a user material subroutine UMAT for the FEM program ABAQUS/Standard [58]. The implicit integration of the crystal plasticity equations was done following the methodology of the work of Huang and Kysar [59, 60]. The constitutive model was also implemented in a Matlab function [61] for calibration purposes. The constitutive equations were solved using the Newton-Raphson iteration method. For 


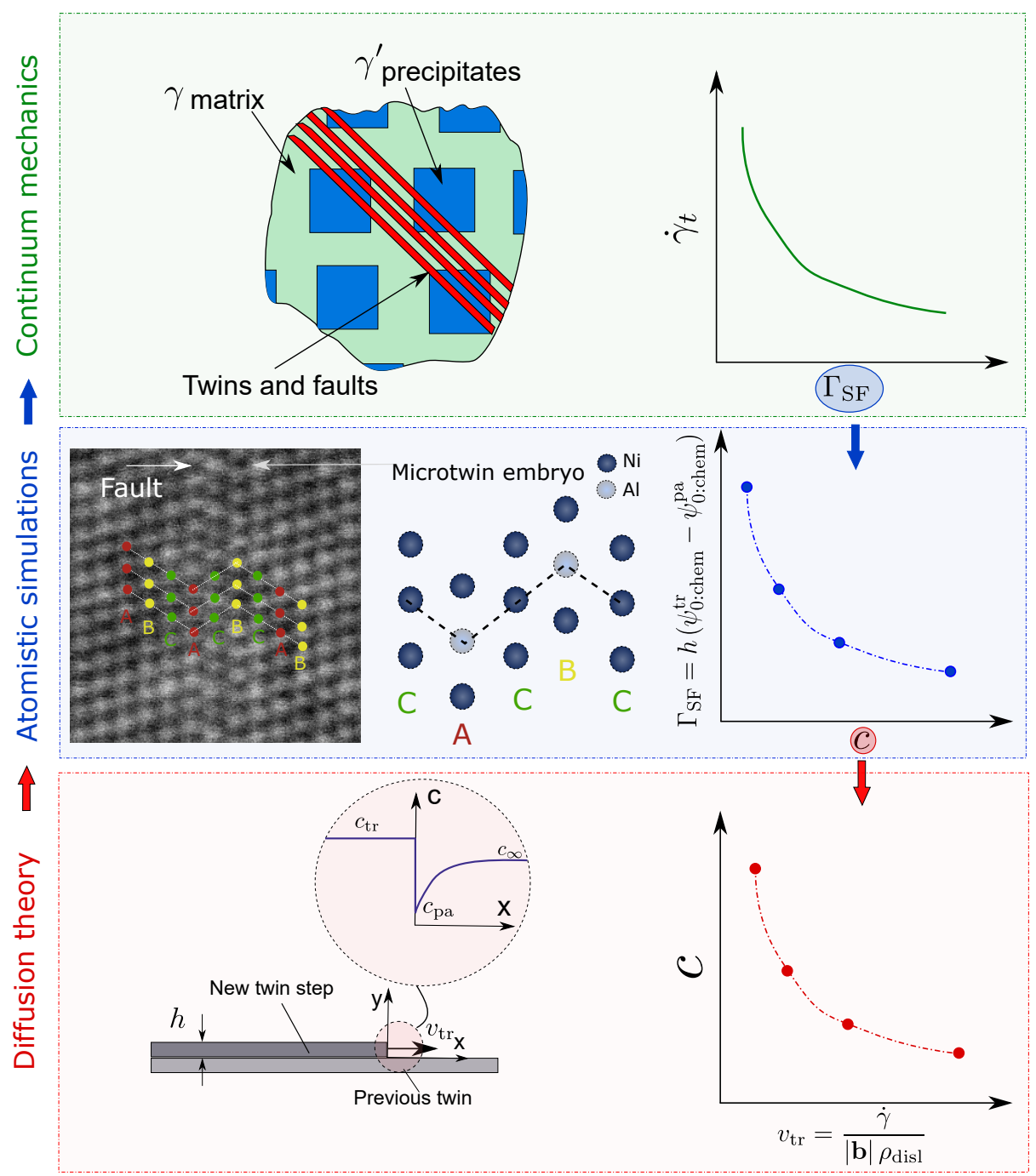

Figure 8: Figure 7: Multiscale diagram of the proposed constitutive model for the diffusionassisted microtwinning formation: (bottom) diffusion theory links growth velocity with Co and $\mathrm{Cr}$ concentration at the twin 4; (center) concentration is associated with stacking fault energy of growing fault using atomistic simulations from the literature [34; (top) finally, stacking fault energy (or equivalently free energy) is connected with macroscopic strain rate. TEM image from Barba et al. [8]. 
each Newton-Raphson iteration, the strain-rate evaluation of the microtwinning

The calibrated model results for $\langle 001\rangle,\langle 011\rangle$ and $\langle 111\rangle$ compression tests are shown in Fig. 9. The elastic and geometrical constants and the calibrated model parameters for the three deformation modes considered are presented in Table 5. More details are given in Appendix D. The application of the model to predict the plastic behavior of single crystals is presented next.

\subsection{Macroscopic creep behavior}

Numerical models with the dimensions of the specimens employed in the uniaxial compression and tension tests described in Section 2 were developed in ABAQUS/Standard. The models were meshed with C3D8 elements. The boundary conditions of the experimental setup were reproduced accordingly. The specific crystal orientations used in the models were extracted from the EBSD measurements and are given in Table 2.

The measured and predicted creep strain-time curves for the tension creep tests along $\langle 001\rangle,\langle 011\rangle$ and $\langle 111\rangle$ are shown in Fig. 9. In all three tensile crystal directions, the macroscopic responses were predicted with reasonable accuracy. For the testing directions where microtwinning was active $(\langle 001\rangle$-compression and $\langle 011\rangle$-tension) the model underestimates the plastic strain of the final stage of the creep test. This can be explained in terms of specific damage mechanisms (not accounted for in the model) associated with the microtwin boundaries where an embrittlement of the samples developing tensile twins is reported [4, 62. In the present work, the same phenomenon is observed for the case $\langle 001\rangle-$ compression testing when microtwinning is activated, leading to final stage with accelerated strain rates, see Fig. 9. These phenomena were rationalized previously in Barba et al. [4]: an increase of dislocation densities in the parent 

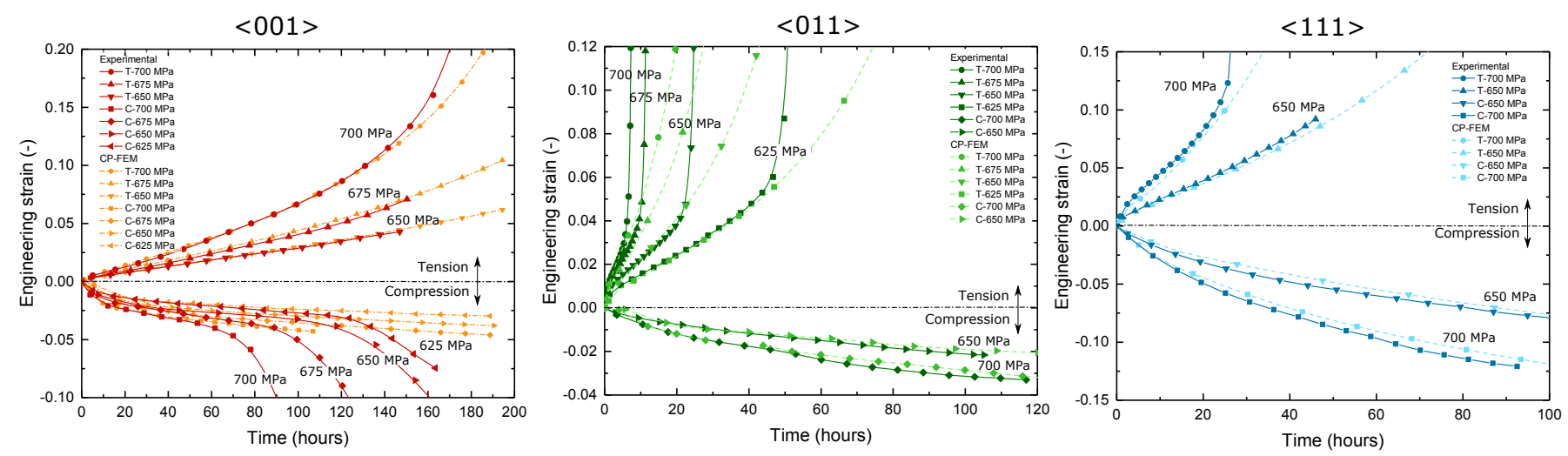

Figure 9: Strain-time experimental curves of MD2-single crystal superalloy at $800{ }^{\circ} \mathrm{C}$ for different stresses vs. model predictions. 
Table 5: Crystal plasticity constitutive parameters for the MD2 Ni-based superalloy. The parameters marked with an asterisk * have been optimised using the creep response of the material while the rest have been obtained from the literature or geometric considerations.

\begin{tabular}{|c|c|c|c|c|c|c|c|c|}
\hline Elastic Properties & $\begin{array}{c}C_{11}(\mathrm{GPa}) \\
23032\end{array}$ & $\begin{array}{c}C_{22}(\mathrm{GPa}) \\
150[32\end{array}$ & $\begin{array}{c}C_{44}(\mathrm{GPa}) \\
9032\end{array}$ & $\begin{array}{c}\gamma_{\operatorname{tr}: \beta}(-) \\
\sqrt{2} / 2\end{array}$ & $\begin{array}{c}\mathrm{h}(\mathrm{m}) \\
7 / 3 \sqrt{3} \times 10^{-10}\end{array}$ & & & \\
\hline Octahedral Slip & $\begin{array}{c}\gamma_{0}^{*}(1 / \mathrm{s}) \\
1.68 \times 10^{-8}\end{array}$ & $\begin{array}{c}m^{*}(-) \\
0.098\end{array}$ & $\begin{array}{c}s_{0}^{*}(\mathrm{MPa}) \\
235\end{array}$ & $\begin{aligned} \tau_{s}^{*} & (\mathrm{MPa}) \\
& 237\end{aligned}$ & $\begin{array}{c}h_{0}^{*}(\mathrm{MPa}) \\
949\end{array}$ & $\begin{array}{c}f_{i j}^{*}(-) \\
1.04\end{array}$ & $\begin{array}{c}\gamma_{0}^{*}(-) \\
0.02\end{array}$ & $\begin{array}{l}q^{*}(-) \\
2.51\end{array}$ \\
\hline Cubic Slip & $\begin{array}{l}\gamma_{0}^{*}(1 / \mathrm{s}) \\
5.7 \times 10^{-7}\end{array}$ & $\begin{array}{c}m^{*}(-) \\
0.098\end{array}$ & $\begin{array}{c}s_{0}^{*}(\mathrm{MPa}) \\
302\end{array}$ & $\begin{aligned} \tau_{s}^{*} & (\mathrm{MPa}) \\
& 290\end{aligned}$ & $\begin{array}{c}h_{0}^{*}(\mathrm{MPa}) \\
951\end{array}$ & $\begin{array}{c}f_{i j}^{*}(-) \\
1.04\end{array}$ & $\begin{array}{c}\gamma_{0}^{*}(-) \\
0.02\end{array}$ & $\begin{array}{l}q^{*}(-) \\
2.51\end{array}$ \\
\hline Twinning & $\begin{array}{c}\gamma_{0}^{*}(1 / \mathrm{s}) \\
1.16 \times 10^{-6}\end{array}$ & $\begin{array}{c}m^{*}(-) \\
0.3\end{array}$ & $\begin{array}{c}s_{0}^{*}(\mathrm{MPa}) \\
52\end{array}$ & $\begin{array}{c}\tau_{s}^{*}(\mathrm{MPa}) \\
76\end{array}$ & $\begin{array}{c}h_{0}^{*}(\mathrm{MPa}) \\
841\end{array}$ & $\begin{array}{c}f_{i j}^{*}(-) \\
1.04\end{array}$ & $\begin{array}{c}\gamma_{0}^{*}(-) \\
0.02\end{array}$ & $\begin{array}{l}q^{*}(-) \\
2.51\end{array}$ \\
\hline
\end{tabular}


phases at the microtwin boundaries was observed. These pile-ups were presumably formed by the abrupt change of Schmid factors between twin and parent phases; the microtwins acted as dislocation barriers. As the parent phase deforms plastically on different slip/twin systems than the principal twin system, the dislocations become increasingly trapped. This damage process could be added in future developments of the constitutive framework presented here. For example, one could limit the amount of plastic deformation that the parent phase can accommodate when the slip and twin systems are different from the main one 63. If this plastic deformation would reaches a critical value, catastrophic failure can occur, as observed experimentally. The increasing strain rates observed in this work in the computational curves are due to the cross-sectional area reduction due to plastic elongation and the fact that both models and experiments are carried out under constant engineering stress.

\subsection{Mechanisms activity}

The activity fraction for each slip (octahedral and cubic) and twin mecha$\operatorname{nism}\left(f_{\text {oct }}, f_{\text {cub }}, f_{\text {twin }}\right)$ is defined as:

$$
f_{i}=\frac{\gamma_{i}}{\gamma_{\text {total }}}
$$

where $i \in\{$ oct,cub,twin $\}$ and $\gamma_{\text {total }}=\gamma_{\text {oct }}+\gamma_{\text {cub }}+\gamma_{\text {twin }}$.

The values obtained for each orientation are plotted in Fig. 10 for $800^{\circ} \mathrm{C}$ and $650 \mathrm{MPa}$ under both tension and compression conditions. The asymmetric behaviors observed in the creep curves correlates well with the type of deformation mechanism activated, especially for $\langle 011\rangle$ and $\langle 001\rangle$ orientations. Twinning is active and responsible for most of the creep plastic deformation for $\langle 011\rangle$-tension and for $\langle 001\rangle$-compression. This is in agreement with previous experimental studies where microtwinning was found to account for most of the plastic de- 
formation during creep (up to $\approx 90 \%$ ) for $\langle 011\rangle$-tension [4, 3, 64]. For $\langle 111\rangle$ specimens, no change of mechanism is found due to the dominant role of cubic slip in this direction and the symmetry of this mechanism. This could explain the reduced asymmetric behavior in this direction when compared with $\langle 001\rangle$ and $\langle 011\rangle$. The activity study presented here was generalized for the whole set of possible single crystal orientations in Fig. 11 for tension and compression cases. Although only the microtwinning mechanism is antisymmetric in terms of its kinematics, the high asymmetry is also extended to the other two mechanisms. The directions in the lower part of the inverse pole figure, along the $\langle 001\rangle-\langle 110\rangle$ pole line present a high asymmetry in terms of activity between tension and compression cases (e.g., the twin and octahedral regions swap). In the upper part, the deformation along the cubic systems is preferred over the two other mechanisms both in tension and compression. The mechanism symmetry also leads to a higher symmetric creep behavior.

Another crucial point regarding the creep deformation of single crystal is the rotation of the lattice during the test, as it has a great influence on the creep life [65, 64, 66, 67]. This rotation is produced when the deformation is limited to only one shear system and the tensile axis is constrained by the loading machine. This phenomenon is defined as 'single slip' 68]. When several slip systems are activated (standard slip or twinning), the rotations of the lattice are less pronounced as the rotations of slip systems counteract each other.

The rotations for the different loading directions are presented in Fig. $12 \mathrm{a}$ for the $800^{\circ} \mathrm{C}-650 \mathrm{MPa}$ cases. The tension and compression cases along the $\langle 111\rangle$ direction and the tension case along the $\langle 011\rangle$ direction show the greatest lattice rotation after testing. This is in agreement with the slip system activities presented in Fig. 12 b for the six different loading conditions. It can be observed that for the cases with large rotations, only one shear system is active. The de- 

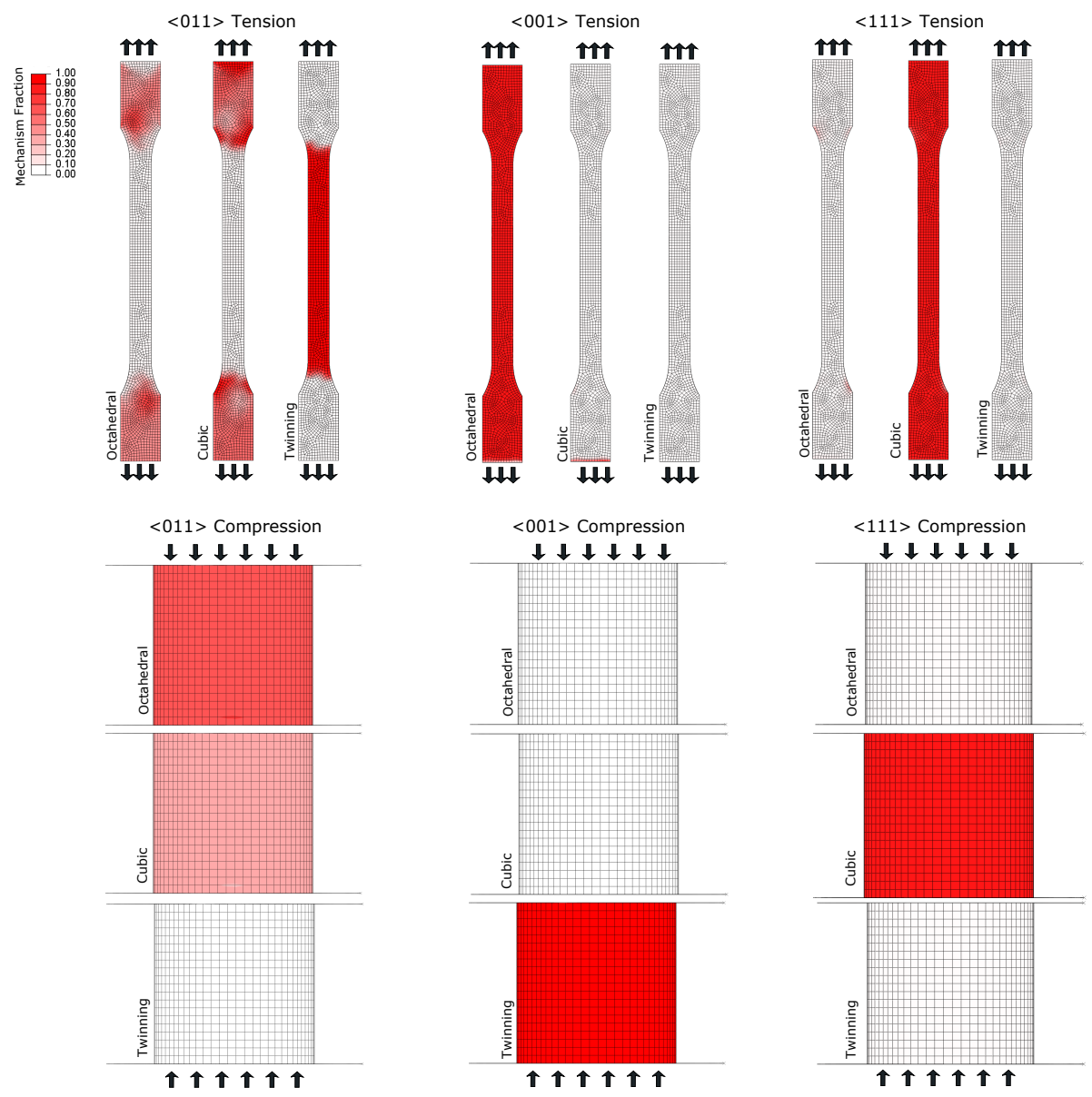

Figure 10: Shear contribution to the total one for each deformation mechanism for simulations performed at $650 \mathrm{MPa}$ and $800^{\circ} \mathrm{C}$. The mechanism fraction is defined as $f_{\text {oct,cub,twin }}=$ $\underline{\gamma_{\text {oct }, \text { cub }, \text { twin }}}$

$\gamma_{\text {total }}$ 


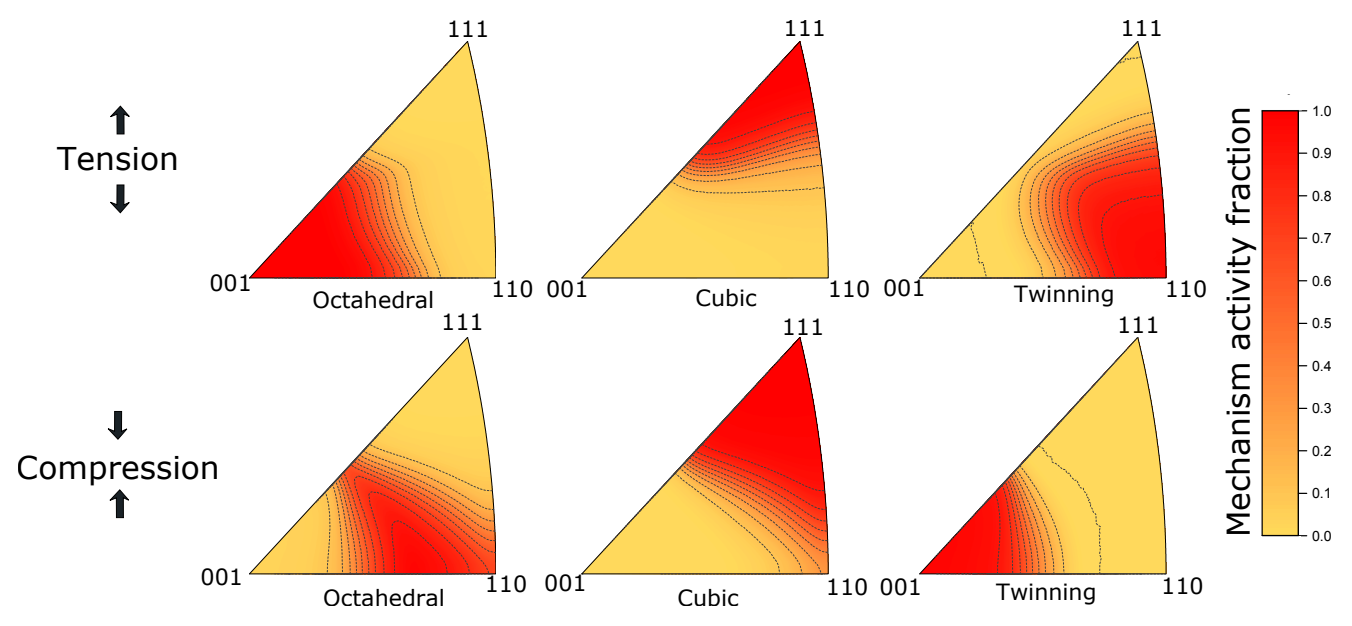

Figure 11: Mechanism activity fraction inverse pole figures for octahedral slip, cubic slip and twinning for both tension and compression cases at $650 \mathrm{MPa}$ and $800^{\circ} \mathrm{C}$. The activity fraction is defined as: $f_{\text {oct }, \text { cub }, \text { twin }}=\frac{\gamma_{\text {oct }, \text { cub }, \text { twin }}}{\gamma_{\text {total }}}$ 
formation is constrained to shear along (111)-[112] twin system for the case of $\langle 011\rangle$ in tension and (100)-[011] for the case $\langle 111\rangle$ in tension and compression. For the tension cases, the loading axis rotates towards the single slip direction while for the compression case, it rotates towards the single slip plane normal, in agreement with $\mathrm{CP}$ theory [68. The time at which $2 \%$ of plastic strain is experimentally reached for each orientation (see Fig. 10 is shown in Table 6 . The time-to-rupture is in agreement with other creep orientation dependence studies 69. This and Fig. 10 indicate a correlation between the single slip cases and the conditions with higher creep rates ( $\langle 111\rangle$ tension and compression and $\langle 011\rangle$ tension). This correlation can also be extended to the time to rupture for the tension cases in Fig. 9. These observations are in agreement with previous experimental studies showing that conditions in which only one deformation shear system is active present the worst creep responses and are thus not desirable [1, 15, 65. A physical explanation for this can be proposed by comparing the two testing conditions with microtwins presented in Fig. 3 a $(\langle 110\rangle$-tension and $\langle 001\rangle$-compression). For $\langle 110\rangle$-tension, only one system of twin bands is observed in agreement with the simulations presented in Fig. 12b. In such condition, the shearing distance free path for gliding dislocations is not affected by the formation of new twins as the partial dislocations run parallel to the preformed twins. However, for $\langle 001\rangle$ compression, the twin band network extends along two other twin planes reducing drastically the mean free path of the gliding dislocations and thus hardening considerably the alloy. Therefore, loading cases where more than one system is active, present lower minimum creep rates and higher creep life. This effect is included in the constitutive model by the cross-hardening term between different slip planes. 
a)
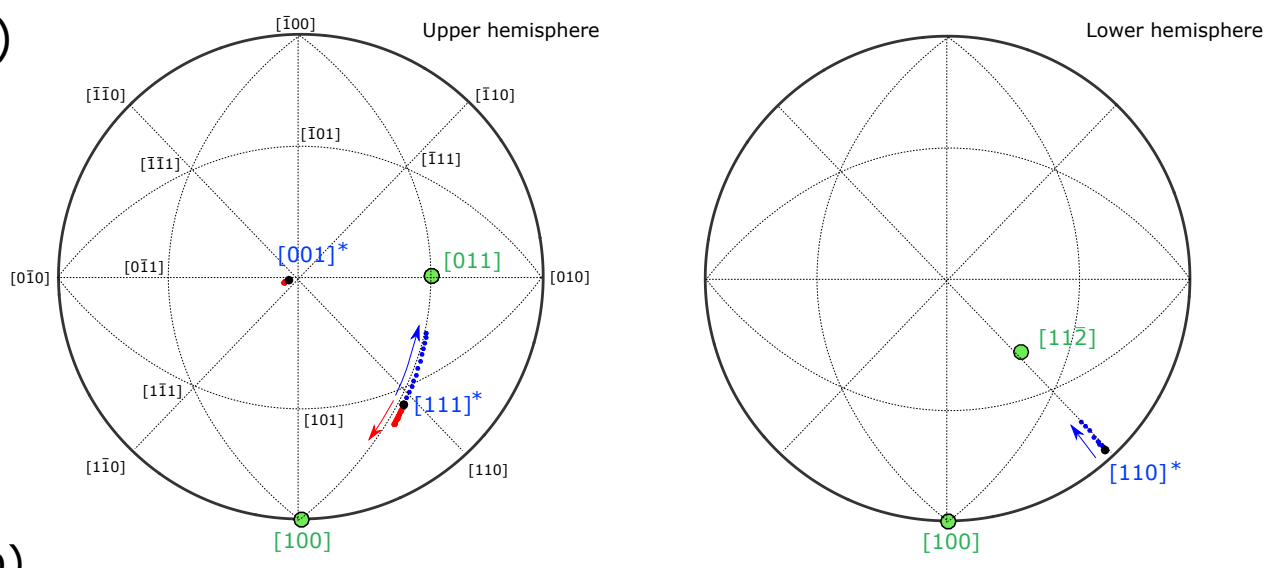

b)
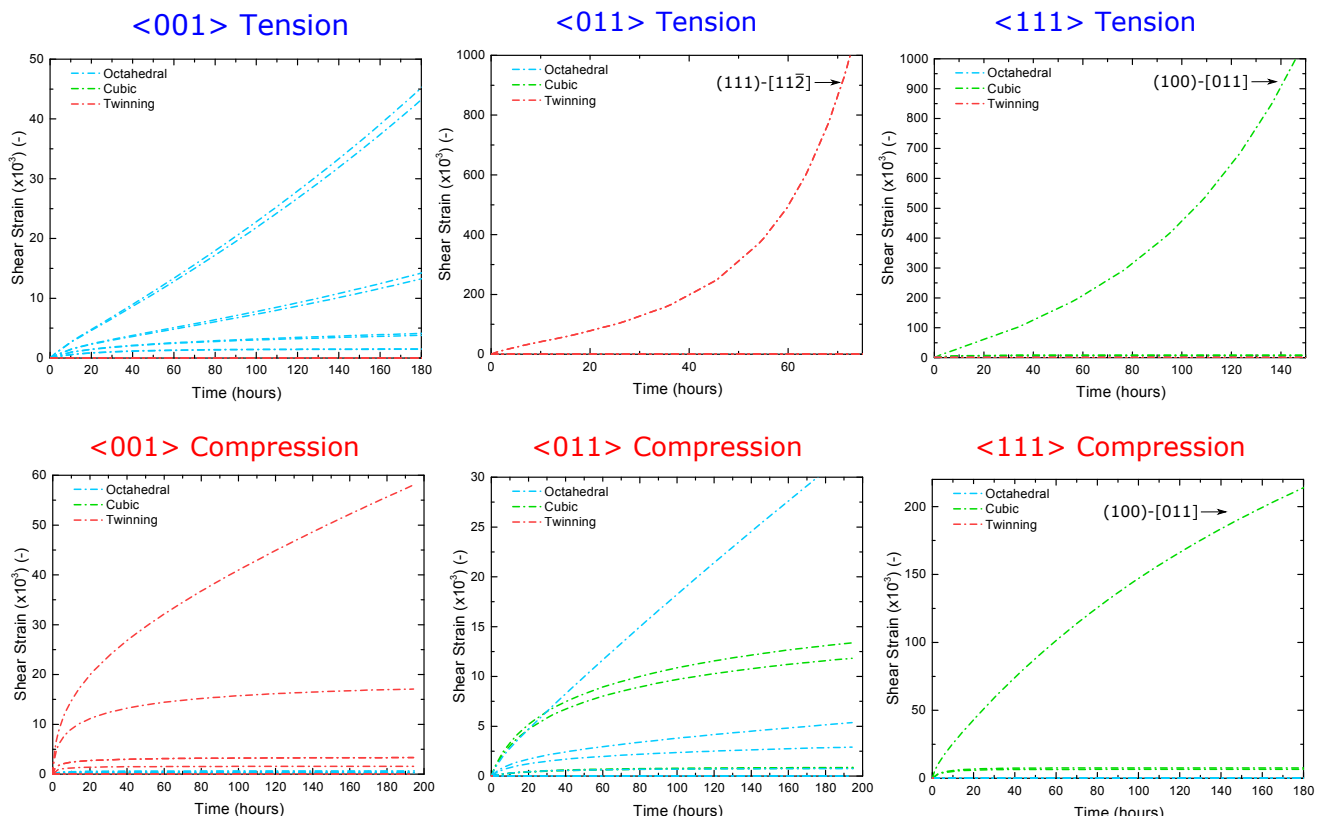

Figure 12: a) Inverse pole figure showing the rotations of the crystal lattice obtained from the single crystal numerical simulations at $800^{\circ} \mathrm{C}$ and $650 \mathrm{MPa}$. In blue the tested crystal orientations and in green the slip direction and normals. The whole orientation plane is shown due to the antisymmetric character of microtwinning; b) shear strains of the different slip systems for the three different tested orientations (red for cubic slip, blue for octahedral slip and green for microtwinning). 
Table 6: Creep times for reaching $2 \%$ plastic strain for each orientation and loading case.

\begin{tabular}{cccc}
\hline & $\langle 001\rangle$ & $\langle 110\rangle$ & $\langle 111\rangle$ \\
\hline Tension & $37.2 \mathrm{~h}$ & $3.2 \mathrm{~h}$ & $6.0 \mathrm{~h}$ \\
\hline Compression & $14.1 \mathrm{~h}$ & $51.3 \mathrm{~h}$ & $3.1 \mathrm{~h}$ \\
\hline
\end{tabular}




\section{Mid-temperatures creep of polycrystalline aggregates}

In this section, the model is used to demonstrate its predictive capability for polycrystalline aggregates. A series of polycrystalline compression creep tests was used to validate the proposed constitutive model. The study is then extended to analyze the tension/compression differences during high temperature deformation.

\subsection{Experimental campaign}

A polycrystalline version with average grain size of $1154 \mu \mathrm{m}$ of the single crystal superalloy MD2 presented in the previous sections is used in this analysis. Cylinders of $5 \mathrm{~mm}$ diameter for $5 \mathrm{~mm}$ length were machined, see Fig. 1 b. A series of compression creep tests at $800^{\circ} \mathrm{C}$ and $650-700 \mathrm{MPa}$ were performed. The tests were run for 150 hours. The strain was measured from the displacement of the grips using non-contact extensometry. An image of the experimental setup is presented in Fig. 13 . Compression tests were selected in this study to minimize grain boundary sliding, the formation of cavities at the grain boundaries and premature failure of the material.

\subsection{Numerical setup}

A numerical model simulating the alloy microstructure was constructed from a series of grain measurements, see Fig. $13 \mathrm{p}$. The measured statistical grain size distributions were fitted to a normal distribution and included in the computational model using the grain-aggregates generator tool Neper [70]. Fig. 13. shows an example of the polycrystalline model used here. The orientation information was extracted from the EBSD measurements of the pre-tested sample and matched with the grain distribution in the computational model.

Compression and tension conditions were both simulated: the former to validate the model against the experimental results and the latter to study 
a)

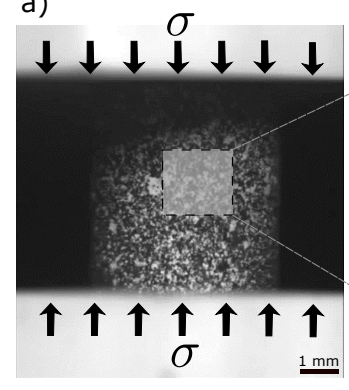

b)

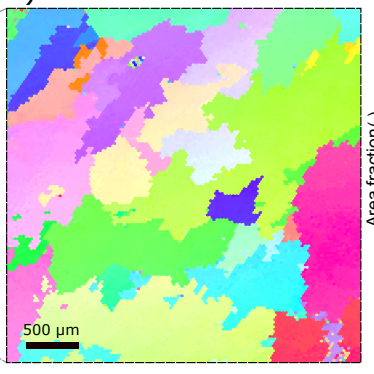

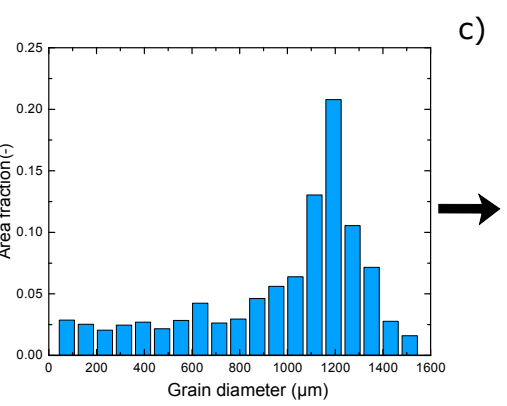

c)
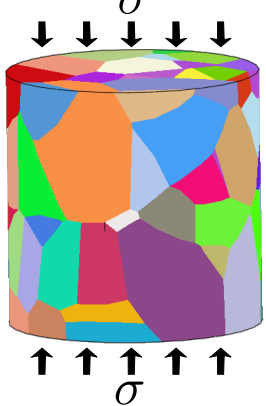

Figure 13: a) Experimental setup for the polycrystalline compression creep tests; b) characteristic EBSD micrograph of polycrystalline MD2 superalloy (left) and statistical grain diameter distribution (right); c) synthetic polycrystalline model used for the numerical simulations. The information obtained from the statistical grain size distribution was used to construct the computational model. 
the tension-compression behavior of polycrystalline superalloys. The boundary conditions for the compression cases were the same as for the single crystal. For the tension cases, the bottom face of the cylinder was fixed in the loading direction and the top was pulled to maintain the coherence with the compression case. The final FEM model contains 103 grains with a total of 5,427 tetrahedral elements and was run using ABAQUS/Standard [58].

\subsection{Mechanical behavior dependence orientation}

The numerical results provided by the polycrystalline model were compared with the experimental data for the compression loading, see Fig. 14. A good correlation was found between the experimental and computational curves, especially in the secondary creep region. The curves slightly differ during the initial primary creep region for $650 \mathrm{MPa}$, but still in the expected scatter of the creep results. This demonstrates the ability of the model to faithfully reproduce the mechanical behavior of polycrystalline materials from single crystal properties.

In Fig. 15a-b, a comparison between the tensile and compression creep cases is presented. In Fig. 15a, simulations exhibit an antisymmetric behavior between tension and compression cases with an inferior creep strength for tension. This is further analyzed in Fig. 15p where the activities of the different mechanisms are computed. The activity is defined as the ratio of shear strain produced by each mechanism to the total one, see Eq. (37). The results show that in most grains, a single deformation mechanism is dominant per grain rather than a mix of several ones. For compression, octahedral and cubic slips are more common than microtwinning, while in tension the contributions of all mechanisms are comparable. The change in mechanism between tension and compression extends along the whole aggregate as observed in the combined activity diagram in Fig. 15b (right). These changes of mechanisms explain the 


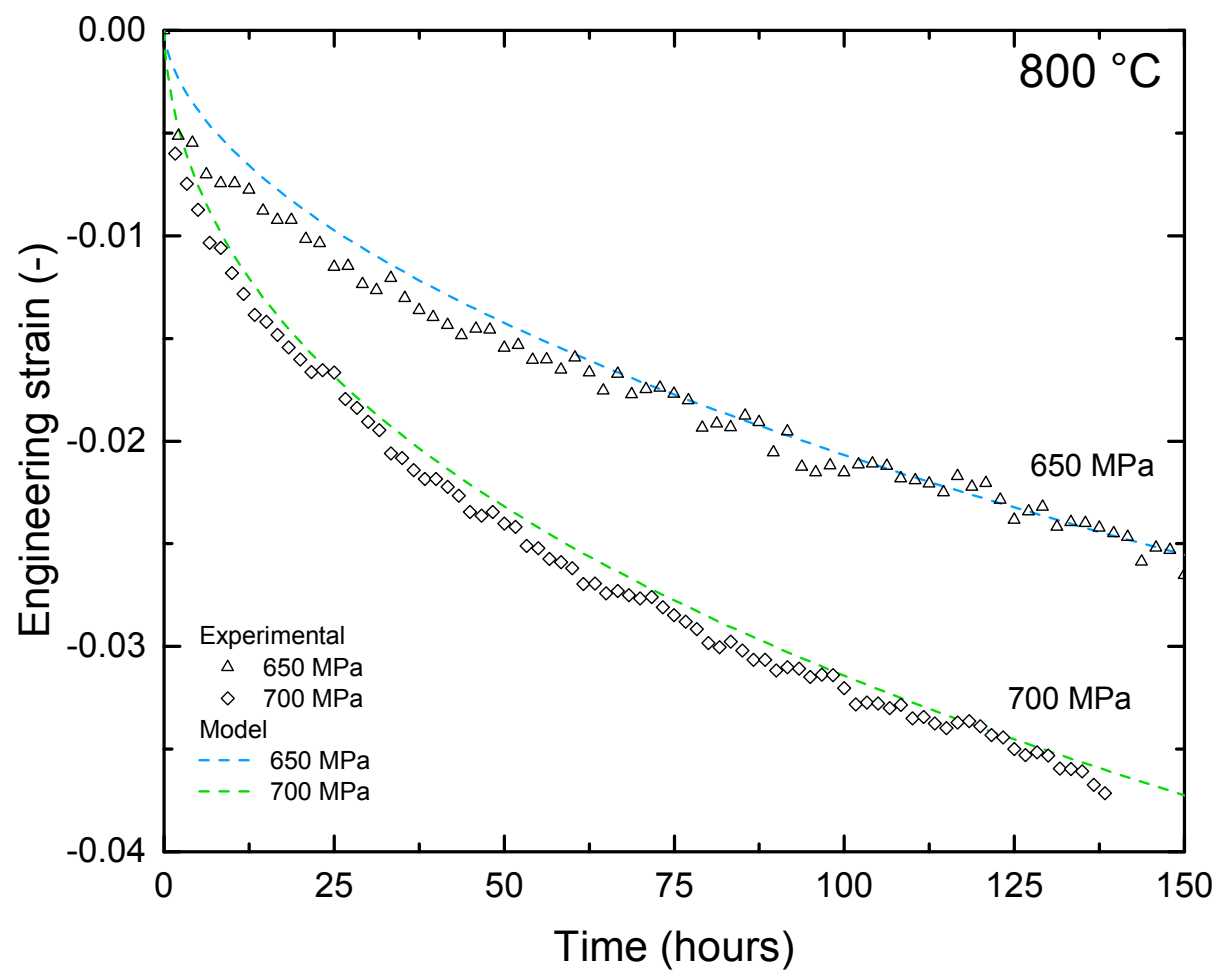

Figure 14: Creep strain vs. time curves used for validation purposes for the polycrystalline superalloy $\mathrm{MD} 2$ tested at $800^{\circ} \mathrm{C}$ under compression stresses of $650 \mathrm{MPa}$ and $700 \mathrm{MPa}$. 
asymmetric strength observed in Fig. $15 \mathrm{p}$ as grains deform by mechanisms with differing creep strengths for the different loading conditions.

The role of the microtwinning mechanism on the creep strength is further supported by several observations linking the formation of microtwins to a premature failure of the alloy [4, 62. In these studies, the local promotion of stress concentrations due to microtwinning was observed to lead to an important damage of the microstructure for single crystal alloys. In the present work, the experimental observations presented in Fig. 15; confirm this phenomenon for polycrystalline alloys. Here, a grain orientation map was obtained from the fracture surface of a MD2 sample after tensile creep testing at $750^{\circ} \mathrm{C}$ and 550 MPa. The deformation marks observed in Fig. 15. (left) at the ending point of the crack surface were analyzed in detail. Here, microtwins were found to extend along grain 1 but not grain 2 . In the former grain, two twin systems are active, one of them parallel to the crack surface. This is in agreement with the grain activity analysis presented in Fig. 15. (right), where the potential active mechanism for each grain was deduced from their Schmid factors. The different active mechanisms for each grain are intimately linked with the crack propagation path. The crack is found to start at the sample surface following the microtwin bands from one of the systems and advancing transgranularly as indicated by the red line. When the crack path encounters the grain boundary in its way to untwinned grain 2 , it changes from transgranular to intergranular. The fact that the crack starts propagating along the microtwinned grains is crucial for the life prediction. This can be due to the lack of strengthening mechanisms in microtwins boundaries, differing from the preformed grain boundaries which are carefully strengthened during the alloying process. In this case, it was found that even though the twinned grains represent only around $\approx 5 \%$ of the total number of grains, their role on the failure mechanism is not 
a)
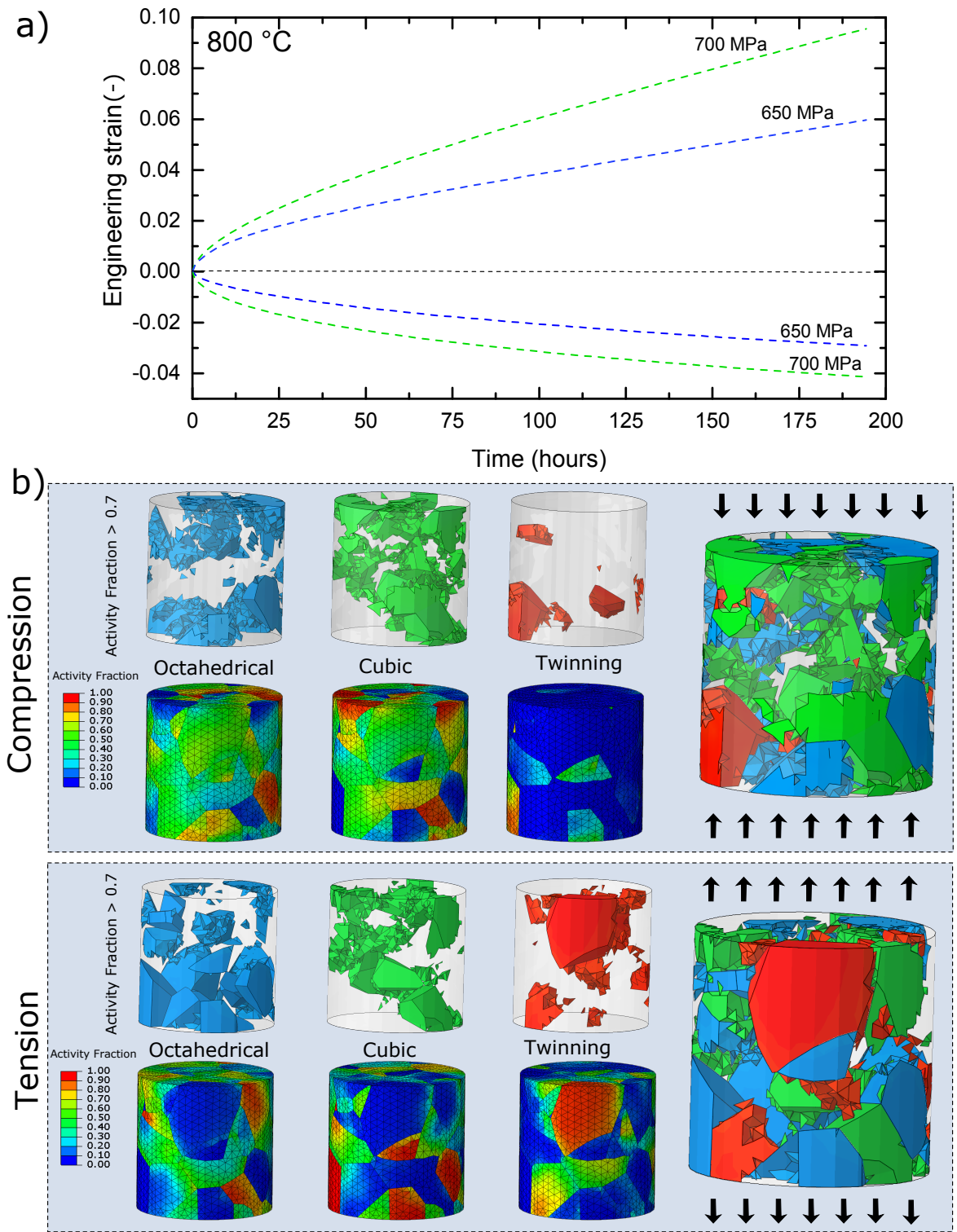

c)

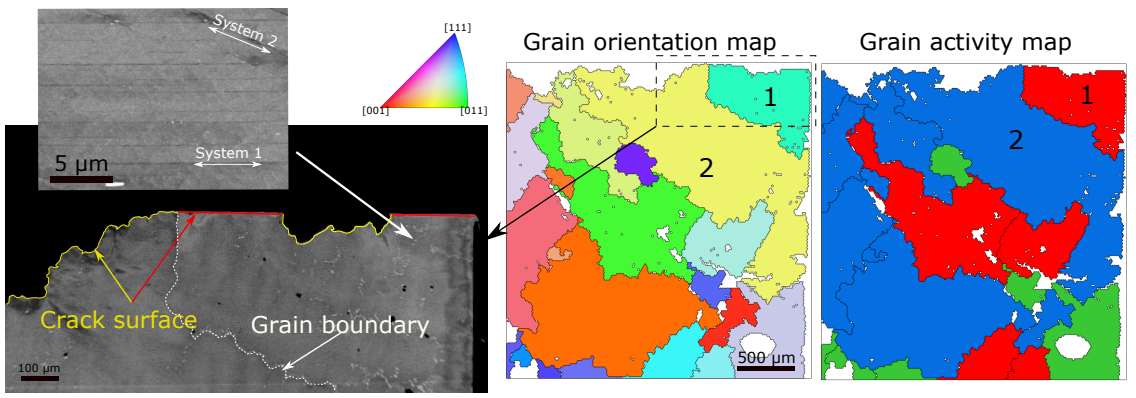

Figure 15: a) Numerical curves for polycrystal simulations at $800^{\circ} \mathrm{C}$ and stresses indicated in tension and compression; b) deformation mechanisms activity analysis for tension and compression simulations under $700 \mathrm{MPa}$ and $800^{\circ} \mathrm{C}$. The mechanism activity is calculated as $f_{\text {oct,cub,twin }}=\frac{\gamma_{\text {oct,cub,twin }}}{\gamma_{\text {toto }}}$; c) fracture surface with microtwins after testing at $750^{\circ} \mathrm{C}$ and tensile stress of $550 \mathrm{MPa}$ (left). Grain orientation map and grain activity map containing the fractured region (right). 
negligible. These results are further proof of the crucial role of microtwinning on the mechanical behavior of Ni-based superalloys at high temperatures.

\section{Conclusions}

This paper analyzes the phenomenon of segregation-assisted plasticity in Ni-based superalloys. The effect was studied experimentally and rationalized using phase-transformation diffusion theory and crystal plasticity theory. The following contributions can be extracted from this work:

- A series of creep tests at $800^{\circ} \mathrm{C}$ on single-crystal superalloy were performed for different stresses and orientations to characterize the mechanical response in this microtwinning temperature regime. A strong tensioncompression asymmetry along the $\langle 001\rangle$ and $\langle 011\rangle$ orientations was found. The post-mortem experimental observations elucidate that microtwinning is active for these directions but only in $\langle 001\rangle$-compression and $\langle 110\rangle$ tension, confirming the asymmetric nature of this mechanism.

- On the basis of these observations, a mechanical constitutive model for Ni-based superalloys was developed accounting for the three main mechanisms observed in these alloys between 700 and $800^{\circ} \mathrm{C}$ : octahedral slip, cubic slip and microtwinning. Based on experimental observations, the microtwinning mechanism was modeled as a phase transformation phenomenon. Thus, the flow rules for the three mechanisms were developed from the driving forces derived from the application of the first and second Thermodynamics Principles. The thermodynamic consistency of the model was been proven.

- The constitutive model was implemented in a FEM code and its parameters were identified for the MD2 single crystal superalloy from experimental data obtained in this paper. A wide range of stress conditions and 
orientations was covered, both in tension and compression states. Good agreement between numerical predictions and experimental results was found in terms of the strain-time curves depending on crystal orientation and stress level. The activation of the different deformation mechanisms correlates well with the ones experimentally observed. A strong connection between the high anisotropy of superalloys and the activation of microtwinnning was found. Moreover, the experimental observations relating elastic rotations during deformation, number of active slip systems and creep life of the specimen were rationalized. The results show that the prevalence of only one active shear system produces large rotations, softens the material due to the lack of hardening mechanisms and thus reduces the creep life of the material.

- The single-crystal constitutive model was extended into a polycrystalline framework in order to study the high temperature behavior of polycrystalline alloys. The numerical creep curves show a good correlation with the experimental tests extending the validation of the model to polycrystalline alloys. Again, a tension-compression asymmetry is observed. The different active mechanisms between tension and compression explain this behavior showing that microtwinning asymmetry cascades into significant changes on the other two mechanisms. Finally, an experimental correlation was found between the active deformation mechanism within a grain and the different crack propagation modes in the grains for polycrystalline alloys. Microtwinning is observed to promote transgranular crack paths and premature initiation of failure, thus reducing the life of the turbine components.

The results presented in this paper demonstrate the capacity of the model proposed to predict the mechanical behavior of Ni-based superalloys at high tem- 
peratures. In addition, the importance of taking into account the coupling between chemistry and plasticity within this temperature range is highlighted.

\section{Appendix A: Expansions of first and second Thermodynamics Prin- ciples}

The reduced global form of energy balance expressed in $\Omega$ is postulated as 71

$$
\frac{\mathrm{d}}{\mathrm{d} t} \int_{\Omega} e \mathrm{~d} V=\int_{\Omega}\left(\boldsymbol{\sigma}: \mathbf{d}-\nabla_{\mathbf{x}} \mathbf{q}+\mathbf{r}\right) \mathrm{d} v
$$

where $e$ is the specific internal energy per unit volume, $\boldsymbol{\sigma}: \mathbf{d}$ is the stress power per unit volume, $\mathbf{q}$ is the heat flow into the body per unit area and $\mathbf{r}$ is the internal heat production, i.e., chemical reactions or radiation, all of these being defined in the current configuration $\Omega$. This energy balance can be rewritten in the reference configuration using $\mathrm{d} V=J \mathrm{~d} V_{0}$ as:

$$
\int_{\Omega_{0}} \dot{e}_{0} \mathrm{~d} V_{0}=\int_{\Omega_{0}}\left(\boldsymbol{\sigma}: \mathbf{d}-\nabla_{\mathbf{x}} \mathbf{q}+\mathbf{r}\right) J \mathrm{~d} V_{0}
$$

where $\dot{e}_{0}$ is the rate of specific internal energy $e_{0}$ per unit reference volume.

The stress power $\boldsymbol{\sigma}: \mathbf{d}$ can be additionally decomposed into the elastic and plastic contributions by using Eq. (4):

$$
\boldsymbol{\sigma}: \mathbf{d}=\boldsymbol{\sigma}: \mathbf{l}^{\mathrm{e}}+\boldsymbol{\sigma}: \mathbf{F}^{\mathrm{e}} \overline{\overline{\mathbf{L}}}^{\mathrm{p}: \mathrm{tr}} \mathbf{F}^{-\mathrm{e}}+\boldsymbol{\sigma}: \mathbf{F}^{\mathrm{e}} \overline{\overline{\mathbf{L}}}^{\mathrm{p}: \mathrm{s}} \mathbf{F}^{-\mathrm{e}}
$$

Here and subsequently, the notations are the same as in the main text. The first term stands for the elastic power; the second term is the contribution of the transformation deformation; and the last term is the standard plastic contribution. Rearranging and expressing each term in its relevant configuration

(dilated configuration $\overline{\bar{\Omega}}$ for slip and phase transformation terms and current 
configuration $\Omega$ for elastic terms), Eq. A3 can be expressed as:

$$
\boldsymbol{\sigma}: \mathbf{d}=J^{-\mathrm{e}}\left(\overline{\overline{\mathbf{M}}}: \overline{\overline{\mathbf{L}}}^{\mathrm{p}: \mathrm{s}}+\overline{\overline{\mathbf{M}}}: \overline{\overline{\mathbf{L}}}^{\mathrm{p}: \operatorname{tr}}\right)+J^{-1}\left(\mathbf{S}_{0}: \mathbf{F}^{T} \mathbf{d}^{\mathrm{e}} \mathbf{F}\right)
$$

The first Thermodynamics Principle can be expressed in its local form in $\Omega_{0}$ by substituting Eq. A4 into Eq. A2]:

$$
\dot{e}_{0}=\left(\overline{\overline{\mathbf{M}}}: \overline{\overline{\mathbf{L}}}^{\mathrm{p}: \mathrm{s}}+\overline{\overline{\mathbf{M}}}: \overline{\overline{\mathbf{L}}}^{\mathrm{p}: \mathrm{tr}}\right) J^{\mathrm{p}}+\mathbf{S}_{0}: \mathbf{F}^{T} \mathbf{d}^{\mathrm{e}} \mathbf{F}
$$

Note that heating effects are neglected assuming constant and uniform temperature $T$ within the body.

The second Thermodynamics Principle reads:

$$
\frac{\mathrm{d}}{\mathrm{d} t} \int_{\Omega} \eta \mathrm{d} V+\int_{\Omega} \nabla_{\mathbf{x}} \frac{\mathbf{q}}{T}-\frac{r}{T} \mathrm{~d} V \geq 0
$$

where $\eta$ is the specific internal entropy per unit of deformed volume. In a similar way to the first Principle, the second Principle can be expressed in the reference configuration $\Omega_{0}$ in its local form as:

$$
\dot{\eta}_{0} \geq 0
$$

As in the first Principle, the heating effects are also neglected here.

\section{Appendix B: Thermodynamic consistency of the model}

The thermodynamic consistency of the phase transformation model is studied here. In order for the process to be fully thermodynamically consistent, the Clausius-Duhem inequality must be satisfied for any arbitrary variation of the state of the system (elastic deformation, slip deformation, phase transformation 
or chemical concentration changes in this case):

$$
\underbrace{J^{\mathrm{p}}\left(\overline{\overline{\mathbf{M}}}: \overline{\overline{\mathbf{L}}}^{\mathrm{p}: \mathrm{s}}\right)}_{\text {slip }}+\underbrace{J^{\mathrm{p}}\left(\overline{\overline{\mathbf{M}}}: \overline{\overline{\mathbf{L}}}^{\mathrm{p}: \text { tr }}\right)+\left(\psi_{0}^{\mathrm{pa}}-\psi_{0}^{\mathrm{tr}}\right) \dot{f}_{0}}_{\text {transformation }}+\underbrace{f_{0}\left(\frac{\partial \psi_{0}^{\mathrm{pa}}}{\partial c_{\mathrm{pa}}}-\frac{\partial \psi_{0}^{\mathrm{tr}}}{\partial c_{\mathrm{tr}}}\right) \dot{c}_{\mathrm{tr}}}_{\text {diffusion }} \geq 0
$$

Following the Coleman and Noll [4] method, we aim at satisfying the positive or

zero condition for each of the terms to assure the thermodynamical consistency of the model.

The first term associated with the slip dissipation can be expressed in the intermediate configuration $\overline{\bar{\Omega}}$ as a function of the slip plastic multipliers $\dot{\gamma}_{\alpha}$ and the projected stress $\tau_{\alpha}$ :

$$
\overline{\overline{\mathbf{M}}}: \overline{\overline{\mathbf{L}}}^{\mathrm{p}: \mathrm{s}}=\sum_{\alpha} \overline{\overline{\mathbf{M}}}:\left(\overline{\overline{\mathbf{m}}}_{\alpha} \otimes \overline{\overline{\mathbf{n}}}_{\alpha}\right) \dot{\gamma}_{\alpha}=\sum_{\alpha} \tau_{\alpha} \dot{\gamma}_{\alpha} \geq 0
$$

where the consistency is obtained implicitly from the flow rule definition in Eq. 28), where the same direction for stress $\tau_{\alpha}$ and the plastic multiplier $\dot{\gamma}_{\alpha}$ is imposed $\left(\tau_{\alpha} \dot{\gamma}_{\alpha} \geq 0\right)$.

Similarly, the second term associated with the transformation dissipation can be rearranged in the intermediate configuration $\overline{\bar{\Omega}}$ using the definition of the plastic flow in Eq. 290, the definition of the projected stress $\tau_{\beta}$ and the relation in Eq. (31) as:

$$
\begin{array}{r}
\overline{\overline{\mathbf{M}}}: \overline{\overline{\mathbf{L}}}^{\mathrm{p}: \operatorname{tr}}+\left(\psi_{0}^{\mathrm{pa}}-\psi_{0}^{\mathrm{tr}}\right) \dot{f}_{0}=\sum_{\beta}\left[\overline{\overline{\mathbf{M}}}:\left(\overline{\overline{\mathbf{b}}}_{\beta} \otimes \overline{\overline{\mathbf{n}}}_{\beta}\right) \gamma_{\mathrm{tr}}+J^{\mathrm{p}}\left(\psi_{0}^{\mathrm{pa}}-\psi_{0}^{\mathrm{tr}}\right)\right] \dot{f}_{0: \beta}= \\
\sum_{\beta}\left[\tau_{\beta}+J^{\mathrm{p}} \frac{\psi_{0}^{\mathrm{pa}}-\psi_{0}^{\mathrm{tr}}}{\gamma_{\mathrm{tr}}}\right] \dot{\gamma}_{\beta} \geq 0 \quad(\mathrm{~B} 3)
\end{array}
$$

where again the consistency is obtained implicitly from the flow rule definition in Eq. (34), where the same direction for the driving force and the plastic 
multiplier $\dot{\gamma}_{\beta}$ is imposed $\left(\left[\tau_{\beta}+J^{-\mathrm{p}} \frac{\psi_{0}^{\mathrm{pa}}-\psi_{0}^{\mathrm{tr}}}{\gamma_{\mathrm{tr}}}\right] \dot{\gamma}_{\beta} \geq 0\right)$.

The third term is associated with the driving force for diffusion between the parent phase and the transformed phase. This is driven by a reduction of the Gibbs free energy per unit volume $\delta G=\delta \psi+\delta p+\eta \delta T$ of the biphasic system, where $\psi$ and $p$, are respectively the Helmholtz free energy and the pressure of the system. In isothermal solid-solid transformations, the term associated with the pressure $p$ is small and can be ignored leading to $\delta \psi=\delta G$. By introducing the definition of the chemical potential $\left(\mu=\frac{\partial G}{\partial c}=\frac{\partial \psi}{\partial c}\right)$, the transformation dissipation term reads:

$$
f_{0}\left(\frac{\partial \psi_{0}^{\mathrm{pa}}}{\partial c_{\mathrm{pa}}}-\frac{\partial \psi_{0}^{\mathrm{tr}}}{\partial c_{\mathrm{tr}}}\right) \dot{c}_{\mathrm{tr}}=f_{0}\left(\mu_{\mathrm{pa}}-\mu_{\mathrm{tr}}\right) \dot{c}_{t r} \geq 0
$$

If we assume a closed system including parent and transformed phase (see Fig. 16) the sign of this term can be expressed as:

$$
\operatorname{sign}(\dot{c})=\operatorname{sign}\left(\left.\delta c\right|_{\delta t}\right)=\operatorname{sign}\left(\left.\delta m\right|_{\delta t}\right)=\operatorname{sign}(-\mathcal{J})
$$

where $\left.\delta c\right|_{\delta t}$ and $\left.\delta m\right|_{\delta t}$ are an infinitesimal concentration increment and a mass increment for an infinitesimal time increment $\delta t$, and $\mathcal{J}$ is the mass flux across the interface of both phases relative to the new phase. Introducing the generalized first Fick's law at the interface $\mathcal{J} \propto\left(\mu_{\mathrm{tr}}-\mu_{\mathrm{pa}}\right)$ [72], the sign of the dissipation term can be expressed as:

$$
\begin{aligned}
& \operatorname{sign}\left(\left(\mu_{\mathrm{pa}}-\mu_{\mathrm{tr}}\right) \dot{c}\right)=\operatorname{sign}\left(\mu_{\mathrm{pa}}-\mu_{\mathrm{tr}}\right) \\
& \operatorname{sign}(-\mathcal{J})= \\
& \operatorname{sign}\left(\mu_{\mathrm{pa}}-\mu_{\mathrm{tr}}\right) \operatorname{sign}\left(\mu_{\mathrm{pa}}-\mu_{\mathrm{tr}}\right) \geq 0
\end{aligned}
$$

which proves that the transformation dissipation term in Eq. B1 is always positive during non-equilibrium $(\dot{c} \neq 0)$ or zero at equilibrium $\left(\mu_{\mathrm{pa}}=\mu_{\mathrm{tr}}\right)$. 


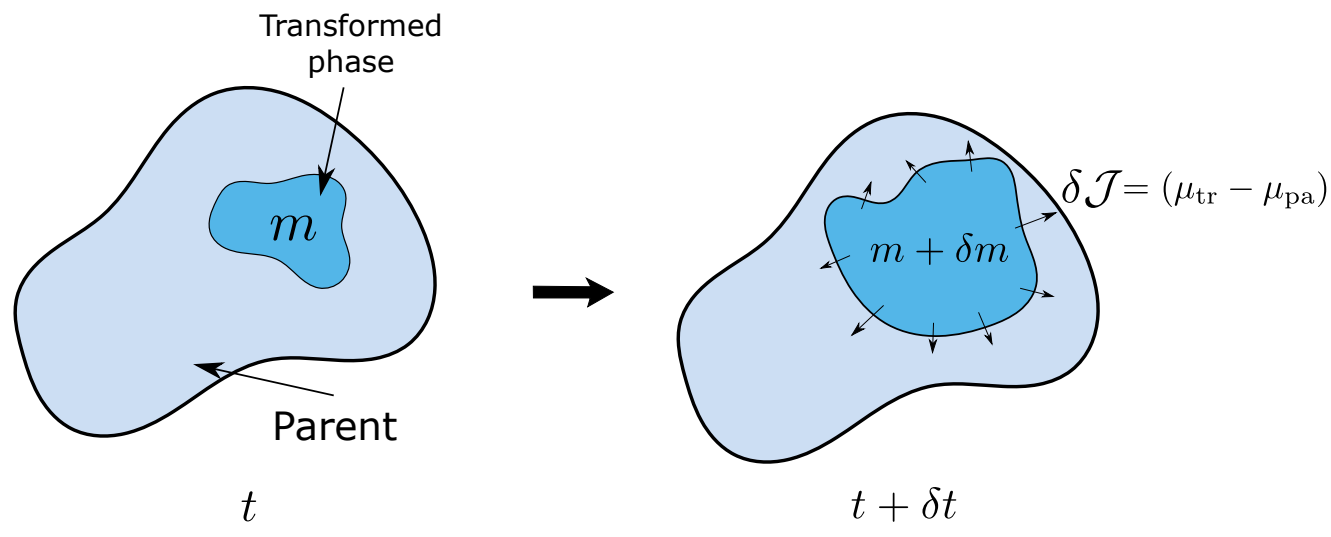

Figure 16: Diagram of the fluxes present in the mass diffusion problem for the case of phase transformation. 


\section{Appendix C: Phase transformation diffusion problem}

The mathematical model proposed by Barba et al. 4] for the case of Co and Cr partitioning controlled growth of microtwins is used here. A schematic of the physical problem is shown in Fig. 55. The boundary condition that governs the ledge growth is obtained from the mass conservation at the interface:

$$
v_{\mathrm{tr}}=\left.D_{\mathrm{eff}} \frac{\partial c}{\partial X}\right|_{\text {step }}\left(c_{\mathrm{tr}}-c_{\mathrm{pa}}\right)
$$

where $v_{\text {tr }}$ is the microtwin ledge velocity, $X$ is the absolute coordinate along the twin length, $D_{\text {eff }}=\frac{D_{\mathrm{Co}} c_{\mathrm{Co}}+D_{\mathrm{Cr}} c_{\mathrm{Cr}}}{c_{\mathrm{Co}}+c_{\mathrm{Cr}}}$ is the effective diffusivity of Co and $\mathrm{Cr}$ in the parent phase and $c_{\mathrm{tr}}$ and $c_{\mathrm{pa}}$ are the effective $\mathrm{Co}+\mathrm{Cr}$ concentrations in the twin phase and in the parent phase at the twin step, respectively.

The diffusion in the parent phase around the twin is governed by Fick's laws [73, whereas for simplicity, the twin phase is assumed to be non-diffusive. The diffusion problem is formulated in a moving adimensional coordinate system $x=\left(X-v_{\text {tr }}\right) / h$ and $y=Y / h$ (relative to global coordinates $\left.X-Y\right)$. Introducing the Péclet number $p=v_{\text {tr }} h / 2 D_{\text {eff }}$, the mathematical problem can be defined as

$$
\left\{\begin{array}{l}
\nabla^{2} \Gamma(x, y)+2 p \frac{\partial \Gamma}{\partial x}=0 \\
\frac{\partial \Gamma}{\partial y}=0 \text { for } y=1, x<0 \\
\frac{\partial \Gamma}{\partial y}=0 \text { for } y=0, x>0 \\
\frac{\partial \Gamma}{\partial x}=-\alpha^{-1}(p) \text { for } x=0,0 \leq y<1
\end{array}\right.
$$

where $\alpha(p)$ is a function to be determined and the dimensionless concentration is defined by

$$
\Gamma(x, y)=\frac{c(x, y)-c_{\infty}}{c_{\mathrm{e}}-c_{\infty}}
$$

where $c_{\mathrm{e}}$ is the equilibrium $\mathrm{Co}+\mathrm{Cr}$ concentration at the interface. The approx- 
imated solution using Fourier transforms and perturbation theory proposed by Trivedi and Jones [74] and Atkinson [75] leads to a set of two non linear equations for $0.003 \leq p<0.500$ :

$$
\left\{\begin{array}{c}
{[\alpha(p)]^{-1}=p+\sum_{i=0}^{7} k_{i}\left[\log _{10}(p)\right]^{i}} \\
c_{0}=2 p \alpha(p)+(p / q)(1-2 p \alpha(p))
\end{array}\right.
$$

where $k_{0}=1.68262, k_{1}=2.84725, k_{2}=5.58040, k_{3}=7.19946, k_{4}=5.49601$, $k_{5}=2.41352, k_{6}=0.55658$ and $k_{7}=0.05182$ and $q=\mu_{0}\left(c_{\mathrm{tr}}-c_{\mathrm{e}}\right)\left(4 D_{\mathrm{eff}} / h\right)^{-1}$ is the non-dimensional interface mobility ( $\mu_{0}$ is the interface mobility). In this system, $\alpha(p)$ and $p$ are the unknowns to obtain and $\Omega_{0}$ is defined as the dimensionless normalized supersaturation concentration $c_{0}=\left(c_{\infty}-c_{\mathrm{e}}\right) /\left(c_{\mathrm{tr}}-c_{\mathrm{e}}\right)$. Once this system is solved, the velocity of the twin ledge can be recovered from the definition of Péclet number. This process allows for the estimation of the microtwin lengthening rate $v_{\operatorname{tr}}$ and, for a kwown characteristic microtwin shear, the specific shear rate $\dot{\gamma}$.

\section{Appendix D: Calibration of the constitutive parameters for MD2 alloy}

The compression creep tests for MD2 superalloy indicated in Table 2 were used here to calibrate the constitutive model. The elastic constants $\left(C_{11}, C_{12}\right.$ and $C_{44}$ ) for MD2 superalloy were obtained from literature data 32. The shear induced by microtwinning $\gamma_{\text {tr }}$ was obtained from geometrical relations [4]. The identification process to obtain the resting plastic model parameters was carried out by inverse optimization approach. To this end, the uniaxial creep straintime curves for compression along $\langle 001\rangle,\langle 011\rangle$ and $\langle 111\rangle$ directions shown in Fig. 2 were used as input data. The optimization problem was solved using a genetic algorithm within the constitutive framework implemented in Matlab 
61 for uniaxial testing conditions. The residual $\mathrm{R}$ is given by

$$
R=\frac{1}{n} \sum_{i=1}^{n}\left(\varepsilon_{i}^{\exp }-\varepsilon_{i}^{\text {num }}\right)^{2}
$$

where $\varepsilon^{\exp }$ and $\varepsilon^{\text {num }}$ represent the experimental and numerical values of the creep strain, respectively. The final stages of deformation were excluded from the optimization process as, during the tertiary creep, the damage mechanisms are dominant and their effect is not included in this model.

Although the proposed constitutive framework has been particularized for the case of MD2 superalloy, the formulation remains general. If another modelled alloy, the whole framework is still valid but further calibration and experimental measurements would be needed. These include:

- Direct atom probe tomography measurements of Co and $\mathrm{Cr}$ (or other) concentrations in the phase and at the microtwins for the given alloy.

- Thermodynamic calculations of the alloy diffusivities using CALPHAD.

- Calibration of the properties indicated with a "*" symbol in Table 5 and the elastic constants $\left(C_{11}, C_{22}\right.$ and $\left.C_{44}\right)$

\section{Acknowledgments}

The authors of this paper are grateful to Jeffrey Fullerton and Stuart Carter for their assistance during the experimental campaign. D.B. acknowledges the EPSRC grant EP/M50659X/1.

\section{References}

[1] R. C. Reed, The superalloys: fundamentals and applications, Cambridge, 2006. 
[2] R. C. Reed, C. M. F. Rae, 22 - Physical metallurgy of the nickel-based superalloys, in: D. E. Laughlin, K. Hono (Eds.), Physical Metallurgy (Fifth Edition), Elsevier, Oxford, 2014, pp. 2215-2290.

[3] T. M. Smith, B. D. Esser, N. Antolin, A. Carlsson, R. E. A. Williams, A. Wessman, T. Hanlon, H. L. Fraser, W. Windl, D. W. McComb, M. J. Mills, Phase transformation strengthening of high-temperature superalloys, Nature Communications 7 (2016) 13434.

[4] D. Barba, D. Pedrazzini, A. Collins, A. J. Wilkinson, M. P. Moody, P. A. J. Bagot, A. Jérusalem, R. C. Reed, On the microtwinning mechanism in a single crystal superalloy, Acta Materialia 127 (2017) 37-40.

[5] V. A. Vorontsov, L. Kovarik, M. J. Mills, C. M. F. Rae, High-resolution electron microscopy of dislocation ribbons in a CMSX-4 superalloy single crystal, Acta Materialia 60 (2012) 4866-4878.

[6] M. Kolbe, The high temperature decrease of the critical resolved shear stress in nickel-base superalloys, Progress in Materials Science A319-321 (2001) 383-387.

[7] L. Kovarik, R. Unocic, J. Li, P. Sarosi, C. Shen, Y. Wang, M. J. Mills, Microtwinning and other shearing mechanisms at intermediate temperatures in Ni-based superalloys, Progress in Materials Science 54 (2009) 839-873.

[8] D. Barba, T. M. Smith, J. Miao, A. Jérusalem, M. J. Mills, R. C. Reed, Segregation-assisted plasticity in ni-based superalloys, 2018. Metallurgical and Materials Transactions A (Under review).

[9] T. M. Smith, R. R. Unocic, H. Deutchman, M. J. Mills, Creep deformation mechanism mapping in nickel base disk superalloys, Materials at High Temperatures 33 (2016) 1-12. 
[10] L. P. Freund, O. M. D. M. Messé, J. S. Barnard, M. Göken, S. Neumeier, C. M. F. Rae, Segregation assisted microtwinning during creep of a polycrystalline L12-hardened Co-base superalloy, Acta Materialia 123 (2017) 295-304.

[11] T. M. Smith, B. D. Esser, N. Antolin, G. B. Viswanathan, T. Hanlon, A. Wessman, D. Mourer, W. Windl, D. W. McComb, M. J. Mills, Segregation and $\eta$ phase formation along stacking faults during creep at intermediate temperatures in a Ni-based superalloy, Acta Materialia 100 (2015) $19-31$.

[12] Q. Qin, J. L. Bassani, Non-schmid yield behavior in single crystals, Journal of the Mechanics and Physics of Solids 40 (1992) 813-833.

[13] N. Tsuno, S. Shimabayashi, K. Kakehi, C. M. F. Rae, R. C. Reed, Tension/compression asymmetry in yield and creep strengths of $\mathrm{Ni}$ based superalloys, Superalloys 2008 (2008) 433-442.

[14] Q. Qin, J. L. Bassani, Non-associated plastic flow in single crystals, Journal of the Mechanics and Physics of Solids 40 (1992) 835-862.

[15] R. N. Ghosh, R. V. Curtis, M. McLean, Creep deformation of single crystal superalloys - modelling the crystallographic anisotropy, Acta Metallurgica et Materialia 38 (1990) 1977-1992.

[16] D. W. MacLachlan, D. M. Knowles, Creep-behavior modeling of the singlecrystal superalloy CMSX-4, Metallurgical and Materials Transactions A 31 (2000) 1401-1411.

[17] S. Keshavarz, S. Ghosh, A. C. E. Reid, S. A. Langer, A non-Schmid crystal plasticity finite element approach to multi-scale modeling of nickel-based superalloys, Acta Materialia 114 (2016) 106-115. 
[18] a. Ma, D. Dye, R. C. Reed, A model for the creep deformation behaviour of single-crystal superalloy CMSX-4, Acta Materialia 56 (2008) 1657-1670.

[19] H. Brehm, U. Glatzel, Material model describing the orientation dependent creep behavior of single crystals based on dislocation densities of slip systems, International journal of plasticity 15 (1999) 285-298.

[20] E. P. Busso, F. T. Meissonnier, N. P. O'Dowd, Gradient-dependent deformation of two-phase single crystals, Journal of the Mechanics and Physics of Solids 48 (2000) 2333-2361.

[21] L. Zhao, N. O’Dowd, E. Busso, A coupled kinetic-constitutive approach to the study of high temperature crack initiation in single crystal nickel-base superalloys, Journal of the Mechanics and Physics of Solids 54 (2006) 288 -309 .

[22] K. Yashiro, M. Naito, Y. Ttomita, Molecular dynamics simulation of edge dislocation piled at cuboidal precipitate in Ni-based superalloy, Transactions of the Japan Society of Mechanical Engineers Series A 69 (2003) $210-217$.

[23] M. Shenoy, Y. Tjiptowidjojo, D. McDowell, Microstructure-sensitive modelling of polycrystalline IN 100, International Journal of Plasticity 24 (2008) 1694-1730.

[24] Y. M. Zhu, A. J. Morton, J. F. Nie, Growth and transformation mechanisms of $18 \mathrm{R}$ and $14 \mathrm{H}$ in $\mathrm{Mg}-\mathrm{Y}-\mathrm{Zn}$ alloys, Acta Materialia 60 (2012) 6562-6572.

[25] Y.-K. Kim, D. Kim, H.-K. Kim, C.-S. Oh, B.-J. Lee, An intermediate temperature creep model for Ni-based superalloys, International Journal of Plasticity 79 (2016) 153-175. 
[26] W. Vorster, F. Dunne, Crystal plasticity and multiscale modelling of superalloy creep, Philosophical Magazine 92 (2012) 830-848.

[27] S. Keshavarz, S. Ghosh, Multi-scale crystal plasticity finite element model approach to modeling nickel-based superalloys, Acta Materialia 61 (2013) 6549-6561.

[28] S. Keshavarz, S. Ghosh, Hierarchical crystal plasticity FE model for nickelbased superalloys: Sub-grain microstructures to polycrystalline aggregates, International Journal of Solids and Structures 55 (2014) 17-31.

[29] S. Keshavarz, S. Ghosh, A crystal plasticity finite element model for flow stress anomalies in $\mathrm{Ni}_{3} \mathrm{Al}$ single crystals, Philosophical Magazine 95 (2015) $1-22$.

[30] P. Kontis, Z. Li, D. M. Collins, J. Cormier, D. Raabe, B. Gault, The effect of chromium and cobalt segregation at dislocations on nickel-based superalloys, Scripta Materialia 145 (2018) 76 - 80.

[31] Planar defect formation in the $\gamma^{\prime}$ phase during high temperature creep in single crystal coni-base superalloys, Acta Materialia 113 (2016) 335-349.

[32] D. Leidermark, J. J. Moverare, S. Johansson, K. Simonsson, S. Sjöström, Tension/compression asymmetry of a single-crystal superalloy in virgin and degraded condition, Acta Materialia 58 (2010) 4986-4997.

[33] D. Barba, S. Pedrazzini, A. Vilalta-Clemente, A. J. Wilkinson, M. P. Moody, P. A. J. Bagot, A. Jérusalem, R. C. Reed, On the composition of microtwins in a single crystal nickel-based superalloy, Scripta Materialia 127 (2017) 37-40.

[34] V. Vorontsov, R. Voskoboinikov, C. Rae, Shearing of precipitates in ni- 
base superalloys: a phase field study incorporating the effective -surface, Philosophical Magazine 92 (2012) 608-634.

[35] D. Bettge, W. Österle, "Cube slip" in near-[111] oriented specimens of a single-crystal nickel-base superalloy, Scripta Materialia 40 (1999) 389-395.

[36] D. Nouailhas, G. Cailletaud, Tension-torsion behavior of single-crystal superalloys: Experiment and finite element analysis, International Journal of Plasticity 11 (1995) 451-470.

[37] T. Tinga, W. A. M. Brekelmans, M. G. D. Geers, Cube slip and non-schmid effects in single crystal ni-base superalloys, Modelling and Simulation in Materials Science and Engineering 18 (2010) 015005.

[38] S. L. Wong, M. Madivala, U. Prahl, F. Roters, D. Raabe, A crystal plasticity model for twinning- and transformation-induced plasticity, Acta Materialia 118 (2016) 140-151.

[39] L. Schemmann, S. Zaefferer, D. Raabe, F. Friedel, D. Mattissen, Alloying effects on microstructure formation of dual phase steels, Acta Materialia 95 (2015) 386-398.

[40] P. C. W. Davies, L. Demetrius, J. A. Tuszynski, Cancer as a dynamical phase transition, Theoretical biology \& medical modelling 8 (2011) 30 .

[41] S. Budday, P. Steinmann, E. Kuhl, The role of mechanics during brain development, Journal of the Mechanics and Physics of Solids 72 (2014) 75-92.

[42] S. R. Kalidindi, C. A. Bronkhorst, L. Anand, Crystallographic texture evolution in bulk deformation processing of fcc metals, Journal of the Mechanics and Physics of Solids 40 (1992) 537-569. 
[43] A. M. Cuitino, M. Ortiz, Computational modelling of single crystals, Materials Science and Engineering 1 (1993) 225-263.

[44] B. D. Coleman, M. E. Gurtin, Thermodynamics with internal state variables, The Journal of Chemical Physics 47 (1967) 597-613.

[45] P. Van Houtte, Simulation of the rolling and shear texture of brass by the Taylor theory adapted for mechanical twinning, Acta Metallurgica 26 (1978) 591-604.

[46] J. P. Hirth, Thermodynamics of stacking faults, Metallurgical Transactions 1 (1970) 2367-2374.

[47] J. R. Rice, Inelastic constitutive relations for solids: An internal-variable theory and its application to metal plasticity, Journal of the Mechanics and Physics of Solids 19 (1971) 433-455.

[48] U. Kocks, A. Argon, M. Ashby, Thermodynamics and kinetics of slip, number v. 19 in International Series of Monographs in Natural Philosophy, Pergamon Press, 1975.

[49] L. Méric, G. Cailletaud, Single crystal modeling for structural calculations: part 2-finite element implementation, Journal of Engineering Materials and Technology 113 (1991) 171-182.

[50] C. Mareau, M. R. Daymond, Micromechanical modelling of twinning in polycrystalline materials: Application to magnesium, International Journal of Plasticity 85 (2016) 156-171.

[51] E. P. Busso, D. W. Dean, D. Linkens, On the effects of loading conditions and geometry on time-dependent singular crack tip fields, Engineering Fracture Mechanics 50 (1995) $231-247$. 
[52] D. Deka, D. S. Joseph, S. Ghosh, M. J. Mills, Crystal plasticity modeling of deformation and creep in polycrystalline ti-6242, Metallurgical and Materials Transactions A 37 (2006) 1371-1388.

[53] M. Samal, S. Ghosh, Evaluation of creep deformation and mechanical properties of nickel-based superalloys through fe analysis based on crystal plasticity models, Procedia Engineering 55 (2013) 342 - 347. 6th International Conference on Creep, Fatigue and Creep-Fatigue Interaction.

[54] J. D. Clayton, A continuum description of nonlinear elasticity, slip and twinning, with application to sapphire, Proceedings of the Royal Society A: Mathematical, Physical and Engineering Science 465 (2009) 307 LP 334.

[55] M. Ardeljan, I. J. Beyerlein, M. Knezevic, Effect of dislocation density-twin interactions on twin growth in az31 as revealed by explicit crystal plasticity finite element modeling, International Journal of Plasticity 99 (2017) 81 101.

[56] Y. Tadano, Y. Yoshihara, S. Hagihara, A crystal plasticity modeling considering volume fraction of deformation twinning, International Journal of Plasticity 84 (2016) $88-101$.

[57] J. L. Bassani, T.-Y. Wu, Latent hardening in single crystals II. analytical characterization and predictions, Proceedings of the Royal Society of London. Series A: Mathematical and Physical Sciences 435 (1991) 21-41.

[58] Abaqus/Implicit, Dassault Systémes, 2010.

[59] Y. Huang, A user-material subroutine incorporating single crystal plasticity in the ABAQUS finite element program, Mech Report 178, Technical 
Report, Division of Engineering and Applied Sciences, Harvard University, Cambridge, Massachusetts, 1991.

[60] J. W. Kysar, Addendum to 'A user-material subroutine incorporating single crystal plasticity in the ABAQUS finite element program, Mech Report 178, Technical Report, Division of Engineering and Applied Sciences, Harvard University, Cambridge, Massachusetts., 1997.

[61] MATLAB, The MathWorks Inc., Natick, MA, 2000.

[62] J. J. Moverare, S. Johansson, R. C. Reed, Deformation and damage mechanisms during thermal-mechanical fatigue of a single-crystal superalloy, Acta Materialia 57 (2009) 2266-2276.

[63] A. Jérusalem, M. Dao, S. Suresh, R. Radovitzky, Three-dimensional model of strength and ductility of polycrystalline copper containing nanoscale twins, Acta Materialia 56 (2008) 4647-4657.

[64] M. G. Ardakani, M. McLean, B. A. Shollock, Twin formation during creep in single crystals of nickel-based superalloys, Acta Materialia 47 (1999) 2593-2602.

[65] C. M. F. Rae, N. Matan, R. C. Reed, The role of stacking fault shear in the primary creep of [001] - oriented single crystal superalloys at $750^{\circ} \mathrm{C}$ and $750 \mathrm{MPa}$, Materials Science and Engineering A 300 (2001) 125-134.

[66] K. Kakehi, Tension/compression asymmetry in creep behavior of a ni-based superalloy, Scripta Materialia 41 (1999) $461-465$.

[67] T. Inoue, K. Tanaka, H. Adachi, K. Kishida, N. L. Okamoto, H. Inui, T. Yokokawa, H. Harada, Evolution of orientation distributions of and phases during creep deformation of ni-base single crystal superalloys, Acta Materialia 57 (2009) 1078 - 1085. 
[68] A. Khan, S. Huang, Continuum theory of plasticity, Wiley-Interscience publication, Wiley, 1995.

[69] P. McHugh, R. Mohrmann, Modelling of creep in a ni base superalloy using a single crystal plasticity model, Computational Materials Science 9 (1997) 134 - 140. Selected papers of the Sixth International Workshop on Computational Mechanics of Materials.

[70] R. Quey, P. Dawson, F. Barbe, Large-scale 3D random polycrystals for the finite element method: Generation, meshing and remeshing, Computational Methods Applied to Mechanical Engineering 200 (2011) 1729-1745.

[71] G. A. Holzapfel, Nonlinear solid mechanics: a continuum approach for engineering science, Meccanica 37 (2002) 489-490.

[72] L. Onsager, Irreversible processes., Phys. Rev. 37 (1931) 237-241.

[73] A. Fick, On liquid diffusion, The London, Edinburgh,and Dublin Philosophical Magazine and Journal of Science 10 (1855) 30-39.

[74] G. J. Jones, R. K. Trivedi, Lateral growth in solid-solid phase transformations, Journal of Applied Physics 42 (1971) 4299-4304.

[75] C. Atkinson, The growth kinetics of individual ledges during solid/solid phase transformations, Proceedings of the Royal Society of London (1981) $351-368$. 